\title{
Localized Controlled Delivery of Gemcitabine via Microsol Electrospun Fibers to Prevent Pancreatic Cancer Recurrence
}

\section{Xia, Guanggai}

2018-09-19

Xia , G , Zhang , H , Cheng , R , Wang , H, Song , Z, Deng , L, Huang , X, Santos , H A \& Cui , W 2018 , ' Localized Controlled Delivery of Gemcitabine via Microsol Electrospun Fibers to Prevent Pancreatic Cancer Recurrence ' , Advanced Healthcare Materials , vol. 7 , no. 18 , 1800593 . https://doi.org/10.1002/adhm.201800593

http://hdl.handle.net/10138/327331

https://doi.org/10.1002/adhm.201800593

acceptedVersion

Downloaded from Helda, University of Helsinki institutional repository.

This is an electronic reprint of the original article.

This reprint may differ from the original in pagination and typographic detail.

Please cite the original version. 


\section{Advanced Healthcare Materials \\ Localized Controlled Delivery of Gemcitabine via Microsol Electrospun Fibers to Prevent Pancreatic Cancer Recurrence \\ --Manuscript Draft--}

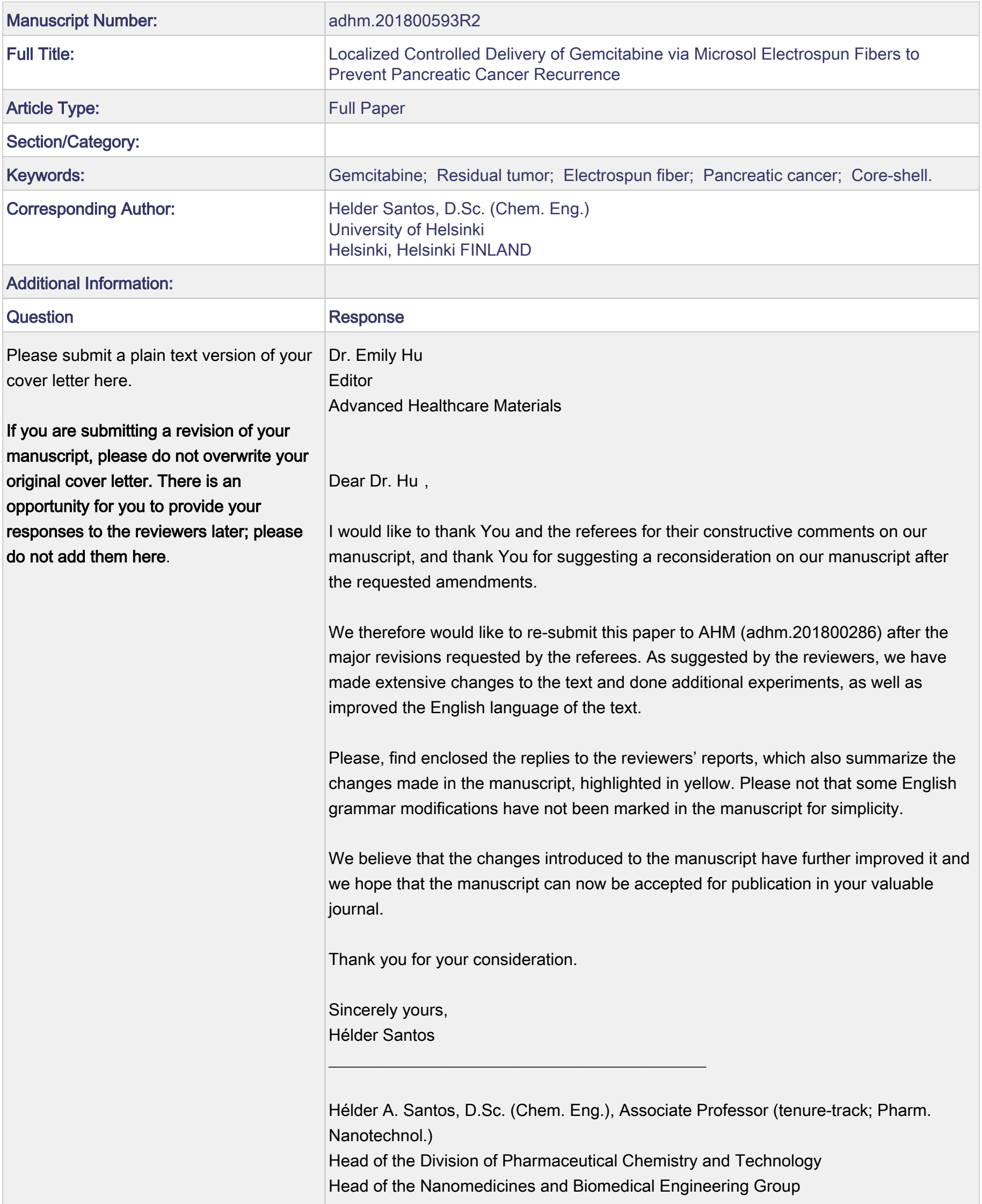




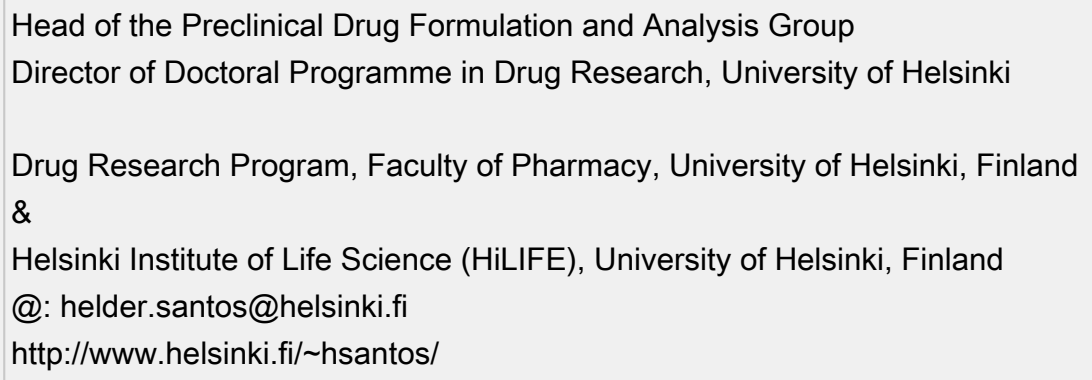

Do you or any of your co-authors have a No. The authors declare no conflict of interest. conflict of interest to declare?

Corresponding Author Secondary Information:

Corresponding Author's Institution:

Corresponding Author's Secondary Institution:

First Author:

$$
\text { Guanggai Xia }
$$

First Author Secondary Information:

Order of Authors:

\begin{tabular}{|l}
\hline Guanggai Xia \\
\hline Hongbo Zhang \\
\hline Ruoyu Cheng \\
\hline Hongcheng Wang \\
\hline Ziliang Song \\
\hline Lianfu Deng \\
\hline Xinyu Huang \\
\hline Helder Santos, D.Sc. (Chem. Eng.) \\
\hline Wenguo Cui \\
\hline
\end{tabular}

Order of Authors Secondary Information:

Abstract:

The low radical surgery rate of pancreatic cancer leads to increased local recurrence and poor prognosis. Gemcitabine (GEM) is the preferred chemotherapeutic for pancreatic cancer. However, systemic chemotherapy with GEM has reached a bottleneck due to its serious side effects after frequent injections. In this study, GEM is successfully enwrapped into electrospun fibers via microsol electrospinning technology to form a stable core-shell fibrous structure. The GEM release rate can be adjusted by altering the thickness of the hyaluronan-sol inner fiber and the quantity of loaded GEM, and the release can be sustained for as long as 3 weeks. In vitro assays show that these electrospun fibers effectively inhibit pancreatic cancer cells and promote apoptosis. In vivo studies show that the fibrous membranes are better for inhibiting the growth of residual tumors than for that of integrated tumors. Furthermore, immunohistochemistry results show that GEM-loaded fibers promote a higher cell apoptosis rate than does systemically injected GEM in residual tumors. In addition, the local delivery of GEM with fibers significantly reduces liver toxicity. In summary, we developed a core-shell electrospun fiber for the controlled and localized delivery of GEM, which greatly improves the treatment of residual tumors and prevents pancreatic tumor recurrence. 


\section{WILEY-VCH}

DOI: $10.1002 /(($ please add manuscript number))

\section{Localized Controlled Delivery of Gemcitabine via Microsol Electrospun Fibers to Prevent Pancreatic Cancer Recurrence}

Guanggai Xia, Hongbo Zhang, Ruoyu Cheng, Hongcheng Wang, Ziliang Song, Lianfu Deng, Xinyu Huang*, Hélder A. Santos* and Wenguo Cui*

Dr. G. Xia, Dr. H. Wang, Dr. Z. Song, Prof. X. Huang.

Department of General Surgery, Shanghai Jiao Tong University Affiliated Sixth People's Hospital, 600 Yishan Rd, Shanghai 200233, P. R. China.

E-mail: 18930177490@163.com;

Dr. R. Cheng, Prof. L. Deng, Prof. W. Cui.

Shanghai Key Laboratory for Prevention and Treatment of Bone and Joint Diseases, Shanghai Institute of Traumatology and Orthopaedics, Ruijin Hospital, Shanghai Jiao Tong University School of Medicine, 197 Ruijin 2nd Road, Shanghai 200025, P. R. China.

State Key Laboratory of Molecular Engineering of Polymers, Fudan University,No.220

Handan Road, Shanghai 200433, China.

Email:wgcui80@hotmail.com

Prof. H. Zhang

Department of Pharmaceutical Sciences Laboratory, Turku Centre for Biotechnology, Åbo Akademi University, 20520, Turku, Finland

Prof. H. A. Santos

Drug Research Program, Division of Pharmaceutical Chemistry and Technology, Faculty of Pharmacy, University of Helsinki, Helsinki FI-00014, Finland

Helsinki Institute of Life Science, HiLIFE, University of Helsinki, FI-00014 Helsinki, Finland Email: helder.santos@helsinki.fi

Keywords: Gemcitabine; Residual tumor; Electrospun fiber; Pancreatic cancer; Core-shell 


\section{WILEY-VCH}

Abstract: The low radical surgery rate of pancreatic cancer leads to increased local recurrence and poor prognosis. Gemcitabine (GEM) is the preferred chemotherapeutic for pancreatic cancer. However, systemic chemotherapy with GEM has reached a bottleneck due to its serious side effects after frequent injections. In this study, GEM is successfully enwrapped into electrospun fibers via microsol electrospinning technology to form a stable core-shell fibrous structure. The GEM release rate can be adjusted by altering the thickness of the hyaluronan-sol inner fiber and the quantity of loaded GEM, and the release can be sustained for as long as 3 weeks. In vitro assays show that these electrospun fibers effectively inhibit pancreatic cancer cells and promote apoptosis. In vivo studies show that the fibrous membranes are better for inhibiting the growth of residual tumors than for that of integrated tumors. Furthermore, immunohistochemistry results show that GEM-loaded fibers promote a higher cell apoptosis rate than does systemically injected GEM in residual tumors. In addition, the local delivery of GEM with fibers significantly reduces liver toxicity. In summary, we developed a core-shell electrospun fiber for the controlled and localized delivery of GEM, which greatly improves the treatment of residual tumors and prevents pancreatic tumor recurrence. 


\section{WILEY-VCH}

\section{Introduction}

Pancreatic ductal adenocarcinoma (PDAC) is one of the most lethal cancers worldwide;

The overall 5-year survival rate is less than $5 \%^{[1]}$. Currently, radical surgery is the most effective way to cure PDAC. Approximately $20 \%$ of PDAC patients can receive surgery when diagnosed $^{[2]}$. However, due to the low rate of negative surgical margins, $80 \%$ of patients experience cancer recurrence within 2 years, which is a notable challenge facing the prognosis of PDAC patients ${ }^{[3]}$.

Adjuvant chemotherapy after surgery decreases the risk of recurrence and improves prognosis in only a small fraction of PDAC patients ${ }^{[4]}$. The main bottleneck is the many disadvantages of anti-tumor drugs, including low bioavailability and strong side effects due to their non-specific biodistribution ${ }^{[2,5]}$. The compactness of cancerous mesenchymal cells in pancreatic cancer also acts as a physiological barrier that prevents drugs from flowing into cancerous parenchymal cells, thereby limiting the effective treatment of PDAC ${ }^{[6]}$. Gemcitabine (GEM), a chemotherapeutic, is one of the best drugs for PDAC patients ${ }^{[7]}$. Anemia, leukopenia, neutropenia, thrombocytopenia, and kidney and liver toxicity are the most common side effects of $\mathrm{GEM}^{[8]}$. As a water-soluble chemotherapeutic agent, GEM primarily relies on intravenous administration, but only a small portion of the drug reaches to the pancreatic cancer through blood circulation ${ }^{[5 b, 9]}$. Since pancreatic cancer has the characteristics of wrapped fibrous tissue and sparse blood vessels within the tumor, drug delivery through blood circulation has low efficient, greatly reducing the ability of the drug to kill pancreatic cancer cells ${ }^{[10]}$. Therefore, developing a more efficient way to enhance the effectiveness of chemotherapeutics and decrease the side effect of drugs to improve the prognosis of pancreatic cancer patients after surgery is urgent.

Polymer-based local drug delivery systems are promising for loading anti-tumor therapeutics for cancer treatment ${ }^{[11]}$. These systems promote the bioavailability of anti-tumor drugs, thereby avoiding non-specific biodistribution, increasing the accuracy of drug targeting, 


\section{WILEY-VCH}

and decreasing the side effects of drugs ${ }^{[12]}$. Particularly, biodegradable polymer-based drugloaded materials can be used to control and sustain drug release over a long period of time ${ }^{[13]}$. For example, electrospinning is a simple technique for producing drug-loaded polymers ${ }^{[14]}$. Electrospun fibers have a larger surface area for promoting drug loading efficiency and a three-dimensional porous structure that can prevent drug diffusion ${ }^{[15]}$. In addition, electrospun fibers are advantageous, because they can be transformed into any shape ${ }^{[16]}$. Therefore, electrospun fibrous membranes can be conveniently implanted into the tumor resection margins for the local delivery of chemotherapeutics and, ultimately, patient treatment.

Water-soluble drugs can be loaded into fibers through emulsion electrospinning, coaxial electrospinning and grafting the drugs through surface functionalization of the fibers ${ }^{32}$. Although emulsion electrospinning has the disadvantage of low drug loading efficiency and a large amount of surfactant addition ${ }^{[17]}$, surface functionalized electrospun fibers can graft water-soluble drugs on the surface of fibers and prevent rapid drug release ${ }^{[18]}$. Coaxial electrospinning can make electrospun fibers with a core-shell structure, and water-soluble drugs can be wrapped in the core of electrospun fibers ${ }^{[19]}$. Another issue with this technique is that the water phase core is instable, therefore drug release is too fast ${ }^{[20]}$. In addition, due to the poor stability of the core-shell structure, adjusting the drug release rate through the internal structure of the core-shell fibers is difficult. Hydrosol is a water-retaining material that fully dissolves water-soluble drugs and creates an interphase to prevent drug diffusion into organic solvents, thereby acting as an excellent drug carrier ${ }^{[21]}$. The combination of emulsion electrospinning and hydrosol for loading water-soluble drugs may achieve high drug loading capacity and controllable drug release with a sustained release profile.

The aim of this study is to further explore a safe, efficient way to improve the survival of PDAC patients after surgery using as a model drug the water-soluble GEM and electrospun fibrous scaffolds comprising poly(L-lactide) (PLA) and hyaluronan (HA, approved by the Food and Drug Administration, FDA) as a model drug. In this study, we apply hydrosol and 


\section{WILEY-VCH}

microsol electrospinning technology to load GEM and develop a GEM-loaded PLA electrospun fibrous membrane, which controls GEM release through changing the thickness of the inner-hydrosol in the fiber. The biocompatibility and drug release behavior of the material are evaluated in vitro, and the cytotoxicity and ability of this material to inhibit pancreatic cancer are investigated in vitro in a nude mouse xenograft tumor and residual tumor models.

\section{Results}

\subsection{Characterization of fibrous scaffolds and GEM Release}

First, we prepared hyaluronic acid hydrosol and used it to dissolve GEM to form GEMHA-sol. Next, GEM-HA-sol was dispersed into PLA solution to form the electrospinning solution through emulsification during which sol particles were dispersed. Finally, solelectrospinning was used to construct the electrospun fibrous membrane with a stable coreshell fibrous structure and loaded with GEM (Figure 1A). We prepared electrospun fibrous membranes containing various amounts of GEM (denoted GEM1@PLA-HA, GEM2@PLAHA and GEM3@PLA-HA) with different contents of sol (denoted GEM1@PLA-HA, GEM1@PLA-HA2, and GEM1@PLA-HA3). Figure 1B shows the morphology of the electrospun fibrous membrane, and no HA-sol particles were observed on the surface of the fiber. The diameters of PLA, GEM1@PLA-HA, GEM2@PLA-HA, GEM3@PLA-HA, GEM1@PLA-HA2 and GEM1@PLA-HA3 fibers were $1.15 \pm 0.38 \mu \mathrm{m}, 1.26 \pm 0.35 \mu \mathrm{m}$, $1.32 \pm 0.46 \mu \mathrm{m}, 1.41 \pm 0.33 \mu \mathrm{m}, 1.30 \pm 0.47 \mu \mathrm{m}$ and $1.21 \pm 0.39 \mu \mathrm{m}$, respectively, and none of the membranes showed significant differences with different quantities of GEM.

The fibrous morphology was further evaluated with transmission electron microscopy (TEM), and a clear core-shell structure was observed for HA-sol-PLA. The inner fibers formed by the HA-microgel had diameters of $0.37 \pm 0.07 \mu \mathrm{m}, 0.48 \pm 0.11 \mu \mathrm{m}, 0.39 \pm 0.09 \mu \mathrm{m}$, $0.30 \pm 0.08 \mu \mathrm{m}$ and $0.24 \pm 0.06 \mu \mathrm{m}$ for GEM1@PLA-HA, GEM2@PLA-HA, GEM3@PLA- 


\section{WILEY-VCH}

HA, GEM1@PLA-HA2 and GEM1@PLA-HA3, respectively. Figure 1B shows that the outer diameter of GEM@PLA-HA was larger than that of PLA, indicating that GEM-sol loading slightly affected the PLA fiber diameter (Table 1). As shown in Table 1, with the same HAsol content, increasing the amount of GEM content did not have a clear effect on the core and shell diameter of the fiber. Both core and shell diameters decreased with a decrease in HA-sol content. Therefore, HA-sol not GEM played a dominant role in affecting the core and shell diameter of the fiber.

The surface wettability of the electrospun membranes was measured by WCA, and the water contact angles (WCA) were $128.3 \pm 2.7^{\circ}, 130.4 \pm 3.3^{\circ}, 127.2 \pm 3.18^{\circ} 128.57 \pm 3.5^{\circ}$,

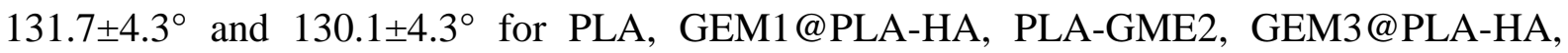
GEM1@PLA-HA2 and GEM1@PLA-HA3, respectively. The wettability of all electrospun membranes revealed a hydrophobic surface, and there were no significant differences among the different groups. Therefore, the fibrous membranes showed poor water permeability.

Subsequently, we examined the release behavior of GEM from the fibers containing different amounts of GEM and HA-sol (Figure 1C). With the increased amount of GEM in HA-sol, the release rate of GEM from the electrospun fibers increased (Figure 1C). With increased GEM loading, the fibrous membrane showed an initial burst release of GEM of 21.4\%, 25.7\% and 29.8\% within 1 day (Figure 1C). GEM3@PLA-HA released GEM over more than 2 weeks, whereas GEM2@PLA-HA and GEM1@PLA-HA released GEM for longer than 3 weeks.

In addition, for the same drug loading content, GEM1, the drug release further decreased by reducing the amount of HA-sol in the fibers. With more HA-sol content in the fibers, the GEM release rate increased (Figure 1C). The initial drug release from GEM1@PLA-HA, GEM1@PLA-HA2 and GEM1@PLA-HA3 was 21.4\%, 19.7\% and 6.8\%, respectively, within 1 day. On the $26^{\text {th }}$ day, the content of GEM released from GEM1@PLA-HA3 was only 2/3 of that released from GEM1@PLA-HA. Electrospun fibers swell in water, and drugs and sol 


\section{WILEY-VCH}

molecules are gradually released from the fiber by diffusion ${ }^{[22]}$. The early burst of drug release was mainly caused by the diffusion of the drug out of HA molecules that created pores in the core. Based on TEM images, GEM1@PLA-HA3 had a thicker shell than did GEM1@PLA-HA $(0.97 \mu \mathrm{m}$ vs $0.89 \mu \mathrm{m})$; this difference in shell thickness might account for the delay of HA and drug diffusion out of the fibers. This result is in good agreement with the findings of a previous study by Wen et al., who showed that increasing the shell thickness can delay the drug release rate ${ }^{[23]}$. Thus, by adjusting the GEM- and HA sol content, it is possible to control GEM release in fibers and tailor them to different kind of biomedical applications.

\subsection{In vitro compatibility of PLA membrane}

Fibroblast cells plays an important role in the progression of pancreatic cancer. These cells promote the proliferation and metastasis of pancreatic cancer cells and can also induce GEM resistance in pancreatic cancer. Hence, we investigated whether a PLA-HA electrospun membrane would affect fibroblast cells and pancreatic cancer cells ${ }^{[24]}$. We first performed a CCK8 assay to investigate cell growth on PLA or PLA-HA electrospun membranes to evaluate whether they were biocompatible. PANC-1 and 3T3 cells (mouse fibroblast cell line) were inoculated on PLA or PLA-HA electrospun membranes in 24-well plates, and cell proliferation was evaluated on days 1 and 3. Comparing with controls, PANC-1 and 3T3 cells grown on electrospun PLA showed no change in cell viability; however, cells grown on HAPLA showed slightly higher viability than did those grown on the control and PLA because HA promotes the adhesive capacity of cells (Figure 2A). This result indicates that HA-PLA and PLA electrospun membranes displayed no cytotoxicity to cells. Furthermore, the adhesive morphologies of 3T3 and PANC-1 cells growth on PLA or PLA-HA electrospun membranes on days 1 and 3 were detected by phalloidin staining to investigate whether the PLA-HA membrane affects fibroblasts or pancreatic cancer cells. We investigated this aspect, because changes in morphology will affect cell function in a certain respect; pancreatic cancer cells 


\section{WILEY-VCH}

change from a polygon shape into a shuttle shape, which promotes metastasis ${ }^{[25]}$. Moreover, and the shuttle shape of fibroblasts might affect the growth rate ${ }^{[26]}$. Figure $2 \mathrm{~B}$ shows that the cell size decreased and the outline of the cells changed when inoculated on PLA or electrospun PLA-HA, which might be due to the three-dimensional structure displayed in the two-dimensional photo. The morphology of fibroblasts remained in a shuttle shape and pancreatic cancer cells remain in a polygonal shape. In addition, no cell morphology change was observed between PLA and PLA-HA in scanning electron microscopy (SEM) (Figure 2C). The above results demonstrate that PLA and PLA-HA electrospun fibrous membranes are biocompatible and can be used for biomedical applications.

\subsection{GEM-loaded PLA-HA membranes in vitro}

The anti-cancer effect of GEM-loaded PLA-HA membranes in vitro was studied by a 3[4, 5-dimethylthiazol-2-yl]-2, 5 diphenyl tetrazolium bromide (MTT) assay. The three GEMloaded PLA-HA membranes contained different quantities of GEM. In Figure 1C, GEM release experiments showed that GEM3@PLA-HA was loaded with the maximum amount of GEM, followed by GEM2@PLA-HA and GEM1@PLA-HA. Figure 3A shows the cell viability of BxPC-3 and PANC-1 cells treated with PLA-control (no GEM-loaded PLA membranes), GEM1@PLA-HA, GEM2@PLA-HA or GEM3@PLA-HA. Compared with the control group, the PLA and PLA-HA groups showed no cytotoxicity when incubated with PANC-1 and BxPC-3 cells. For the other groups, GEM3@PLA-HA showed higher antiproliferation activity than did GEM2@PLA-HA and GEM1@PLA-HA against PANC-1 and BxPC-3 cells $(\mathrm{P}<0.001)$, because GEM3@PLA-HA loaded and released more GEM than did GEM2@PLA-HA and GEM1@PLA-HA. In addition, the PANC-1 cell viability decreased with an increased incubation time with PLA-HA-GEM; cell viability almost decreased to zero at day 7 , the results suggested that the GEM-loaded membranes needed time to release more 


\section{WILEY-VCH}

gemcitabine to effectively kill pancreatic cancer cells. Since GEM induces apoptosis in cancer cells through interfering with DNA replication ${ }^{[27]}$, we performed an Annexin V/propidium iodide (PI) double staining to determine which kind of material possessed the greatest ability to induce cell apoptosis in BxPC-3 and PANC-1 cells. GEM3@PLA-HA had the strongest effect on apoptosis induction, which was in good agreement with the MTT assay (Figure 3B). Thus, cell cytotoxicity was positively associated with the loaded amount of GEM in the fibers and the treatment time, and we selected GEM3@PLA-HA for subsequent in vivo studies.

\subsection{In vivo anti-integrated tumor capability}

A xenograft tumor model of pancreatic cancer was used to evaluate the anti-tumor ability of GEM3@PLA-HA in vivo. We implanted a GEM3@PLA-HA electrospun membrane into the tumor surface as depicted in Figure 4A. The tumor volume and tumor weight of mice that received GEM3@PLA-HA local chemotherapy were almost the same as those of mice that received GEM chemotherapy (GEM group) (Figure 4B-D). Weight change was an easily measured indicator of the side effect of chemotherapy. We noticed that the weights of mice in the GEM group were significantly lower than those of mice in the PLA-GEM3 group (Figure 4E). Systemic administration of GEM caused side effects because of the whole biodistribution of GEM unlike local chemotherapy with PLA-GEM3, which minimized the side effects of the drug, since GEM mainly acted at the tumor site instead of being distributed throughout the whole body.

We also performed immunohistochemical (IHC) staining to evaluate the proliferation and apoptosis status in the tumor tissues of the two groups (Figure 4F). The staining levels of the proliferation index Ki-67 and the anti-apoptosis index Bcl-2 were similar between the two groups, and the pro-apoptosis index cleaved caspase- 3 also exhibited the same tendency (Figure 4G). These results demonstrate that the efficiency of local chemotherapy with a 


\section{WILEY-VCH}

GEM3@PLA-HA electrospun membrane was as good as that of systemic chemotherapy with GEM, while extensively minimizing the side effects of the drug.

\subsection{In vivo anti-residual tumor effect}

Radical surgery for pancreatic cancer is difficult to achieve; therefore, the local tumor recurrence rate after surgery remains high, and compared with a integrated tumor, a residual tumor is often more aggressive and difficult to treat. In this study, we further investigated the anti-residual xenograft tumor capability of GEM3@PLA-HA. The GEM3@PLA-HA electrospun membrane (Figure 5A) was implanted into the residual tumor and compared with systemic chemotherapy with GEM. Interestingly, the anti-tumor capability of GEM3@PLAHA was superior to that of GEM, and compared with the control, GEM also showed an antitumor effect (Figure 5B). As shown in Figure 5C and D, the tumor volume $(\mathrm{P}=0.0188)$ and tumor weight $(\mathrm{P}=0.0134)$ of the GEM3@PLA-HA group were significantly lower than those of the GEM group and control group. The mouse weight of the GEM3@PLA-HA group was higher than that of the GEM group and control group (Figure 6E). Furthermore, we detected the expression of Ki-67, Bcl-2 and cleaved caspase 3 with IHC (Figure 6F). The proliferation index Ki-67 and the anti-apoptosis index Bcl-2 were lower in the GEM3@PLA-HA group, and the apoptosis index cleaved caspase3 was higher in the GEM3@PLA-HA group (Figure 6G). All these results demonstrate that local GEM delivery with PLA-HA was more effective than systemic drug delivery for treating residual tumors when tumor integrity was destroyed. Thus, the deficiency in the protective membrane of the tumor benefited the GEM delivery from the GEM-loaded electrospun membranes.

\subsection{Side effect reduction of GEM-loaded electrospun fibers}

Only a small amount of GEM can enter the tumor tissue when systemic chemotherapy is performed in patients, and most of the GEM is absorbed by normal tissues ${ }^{[10 b]}$. Therefore, 


\section{WILEY-VCH}

GEM leads to serious side effects in pancreatic cancer patients, and liver toxicity is particularly obvious ${ }^{[28]}$. Our study found that GEM-loaded electrospun fibrous membranes reduce the side effects of GEM on liver toxicity. As shown in Figure 6, nude mice receiving systemic chemotherapy with GEM had liver damage and hematoxylin and eosin (HE) staining of the liver showed liver cell necrosis, while no necrosis was observed for the mice receiving GEM3@PLA-HA localized chemotherapy or saline alone(Figure 6A). We also measured blood plasma aspartate aminotransferase (AST) and alanine aminotransferase (ALT), which reflect the liver function of nude mice treated with GEM or GEM3@PLA-HA. The AST and ALT levels of mice in the GEM group were significantly higher than those in the GEM3@PLA-HA group (Figure 6B). These results suggest that the localized delivery of GEM with PLA-HA electrospun membranes to pancreatic cancer could decrease the toxicity of GEM, which is clinically important for the treatment of PDAC.

\subsection{Mechanism for drug release and chemotherapy}

Currently, the most effective treatment for pancreatic cancer is surgery, but only approximately $20 \%$ of pancreatic cancer patients can receive surgery, because most patients are in the advanced stage when diagnosed. However, only $20 \%$ of these patients can receive radical surgery, and the remaining $80 \%$ who undergo pancreatic cancer surgery might have local tumor cell residue ${ }^{[29]}$. Systemic chemotherapy with GEM is a way to improve the survival of pancreatic cancer patients ${ }^{[28,30]}$. Due to its special pathologic characteristics, pancreatic cancer has a poor blood supply; pancreatic cancer cells are surrounded by abundant fibrous connective tissue that compresses tumor blood vessels and cause chemotherapeutics to concentrate; compared with other types of solid tumors, pancreatic tumors exhibit less blood flow into the cancerous tissue through the circulation ${ }^{[10 a, 31]}$. 


\section{WILEY-VCH}

In view of the above situation, we used GEM-loaded PLA-HA electrospun fibrous membranes with a core-shell structure using sol-electrospinning technology to provide sustained release of GEM at the site of the tumor. The release time of GEM could be adjusted by the thickness of core and sustained over 3 weeks. Localized drug delivery has been proven to effectively inhibit the growth of tumors in cervical ${ }^{[12 b]}$, $\operatorname{colorectal}^{[32]}$, and liver cancers ${ }^{[13 b]}$; it also reduces the side effects of chemotherapeutics.

As illustrated in Figure 7, one of the physiological features of PDAC is that the tumor is tightly packed and surrounded with fibrous tissue, which presents a great challenge for drug penetration into tumor ${ }^{[33]}$. Thus, in the xenograft pancreatic tumor model, GEM@PLA-HA did not show better efficiency than systemically administered GEM(Figure 7A). Localized delivery of the drug to a particular body compartment, thereby lowering the systemic drug level, suggesting that localized delivery drug could penetrate to vessels ${ }^{[34]}$. However, the fibrous tissue in pancreatic cancer not only prevented the gemcitabine released by gemcitabine-loaded membranes from penetrating to tumor and tumor vessels, but also compressed the tumor vessels which reduced the concentration of gemcitabine. Therefore, after surgery, with the loss of tumor integrity, the fibrous tissue around the tumor is also destroyed. The destruction of the fibrous matrix in pancreatic cancer could promote GEM penetration into the tumor tissue ${ }^{[10 b, 35]}$. Thus, the gemcitabine locally released from GEMloaded membranes was more capable of penetrating tumor tissues (Figure 7B). GEM@PLAHA was combined with sol-electrospinning technology and a GEM-loaded hyaluronic acid hydrogel, which showed significantly better efficicacy in the treatment of residual tumors than did systemically administered GEM. In addition, when tumor was completely resected, the GEM-loaded membranes could kill the pancreatic cancer cells which might scattered in the surgical site.

PLA-HA electrospun fibers had good biocompatibility, and another great advantage of using it to load GEM is reducing the side effects. The localized and sustained release of GEM 


\section{WILEY-VCH}

minimized the systemic biodistribution of the drug and greatly reduced the spread of GEM to healthy tissues, such as the liver. In our study, mouse bodyweight was clearly higher in the GEM@PLA-HA group than in the GEM group, indicating the reduced side effects of the drug. GEM@PLA-HA also clearly reduced the liver injury and liver toxicity. In addition, it is very easy to attach GEM@PLA-HA to the tumor bed after surgery because the shape of the electrospun fibrous membrane could be tailored. Therefore, for patients with pancreatic cancer after surgery, the membranes could be attached to the local surgical site and continue to release GEM to kill possible residual tumor cells and prevent tumor recurrence. However, GEM-loaded fibrous membranes as a therapeutic tool for localized treatment of pancreatic cancer after surgery also had drawbacks. For example, the membranes must be placed into the surgical field only one time, resulting in limited for long-term localized treatment of pancreatic cancer after surgery. In addition, this kind of localized treatment could not significantly increase the concentration of a chemotherapeutic agent in the blood, thus it might not work well for pancreatic cancer cells that might have already metastasized into the blood circulation, combined with systemic chemotherapy of GEM might help to resolve the deficiency of localized chemotherapy, and they could collaborate to against pancreatic cancer.

\section{Conclusions}

GEM was dispersed in hyaluronic acid hydrosol and subsequently enwrapped into PLAHA electrospun fibers via sol-electrospinning technology to form stable core-shell fiber structures. The GEM loading content can be easily adjusted by dispersing different amounts of GEM in HA sol-gel, and the release rate is controlled by the thickness of the sol-gel core in the PLA fiber. Sustained GEM release lasted over 3 weeks, which facilitated its in vivo application for localized drug delivery. GEM@PLA-HA electrospun fibrous membranes were easily implanted onto the surface of tumors; however, the anti-tumor effect was not superior 


\section{WILEY-VCH}

to GEM treatment in animal models with an integrated xenograft pancreatic cancer tumor, this difference might be due to the fibrous tissue around the tumor limiting GEM penetration into tumor tissues when the tumor was integrated. However, compared with intravenously administered GEM, the GEM@PLA-HA electrospun fibrous membrane was more efficient inhibiting the growth of residual tumors. Furthermore, GEM@PLA-HA significantly reduced the adverse side effects of GEM, including reduced liver toxicity. Therefore, this kind of local implanted drug-loaded scaffold has potential in the prevention and treatment of tumor recurrence after surgery. Overall, in this study, we developed a promising platform of GEM@PLA-HA electrospun fibrous membranes for local chemotherapy of pancreatic cancer.

\section{Experimental Section}

\section{Materials.}

PLA $(\mathrm{Mw}=140 \mathrm{kDa}, \mathrm{Mw} / \mathrm{Mn}=1.85)$ was purchased from Jinan Daigang Co. (Jinan, China). Fermentation-derived HA (sodium salt, $\mathrm{Mw}=0.5 \mathrm{MDa}$ ) was purchased from Yuancheng Technology Co. (Wuhan, China) and used without further purification. GEM was purchased from Eli Lilly and Company (USA). All solvents were purchased from Jiangsu Qiangsheng Functional Chemistry Co., Ltd. and used without further purification.

\section{Fabrication and characterization of electrospun fibrous scaffolds.}

HA hydrosols ( $1 \mathrm{wt}-\%$, obtained by adding $0.1 \mathrm{~g}$ HA to $9.9 \mathrm{~g}$ distilled water and stirring until completely dissolved) were prepared in three groups containing 30, 45 and $60 \mathrm{mg}$ of GEM in $1 \mathrm{~mL}$ HA solution. Drug-loaded HA-sol was added to a solution of dichloromethane (DCM, 8.0 g) containing $1 \%$ sorbitan monooleate (Span-80,with respect to PLA) (Table 1). To prepare uniform water-in-oil (W/O) emulsions containing microsol particles, we stirred the mixture vigorously for $20 \mathrm{~min}$. Next, $1.0 \mathrm{~g}$ PLA and $4.0 \mathrm{~g}$ of N,N-Dimethylformamide (DMF) were added into the emulsion and dissolved entirely to obtain a homogeneous electrospinning 


\section{WILEY-VCH}

solution. PLA solution was used as the control and prepared as follows: $1.0 \mathrm{~g}$ PLA was added to $8.0 \mathrm{~g} \mathrm{DCM}$ and stirred completely, and then $2.0 \mathrm{~g}$ DMF was added to the mixture. The electrospinning equipment had a high voltage statitron (Tianjing High Voltage Power Supply Co., Tianjing, China) set to $10-25 \mathrm{kV}$. The electrospinning solution was added by a $5-\mathrm{mL}$ syringe attached to a metal needle with a $0.6-\mathrm{mm}$ diameter and was electrospun at the flow rate of 0.2 to $6 \mathrm{~mL} / \mathrm{h}$ controlled by a microinject pump (Lange Medical Instrument Co., Baoding, Hebei, China). The GEM-loaded PLA electrospun fibrous membranes were obtained through microsol electrospinning technology. PLA solution was used as the control and prepared as follows: $1.0 \mathrm{~g}$ PLA was added to $8.0 \mathrm{~g}$ of DCM and stirred completely, and $2.0 \mathrm{~g}$ of DMF was added to the mixture. All of the fibrous scaffolds were lyophilized overnight to remove the solvent and water residue and stored at $4{ }^{\circ} \mathrm{C}$ in a desiccator until further use.

Fiber morphologies were examined by field-emission scanning electron microscopy (FESEM) using a Hitachi 4800 system with an acceleration voltage of $3.0 \mathrm{kV}$. Before SEM observation, the fibers were sputter-coated with platinum. TEM images of an individual fiber's inner structure were obtained by a Hitachi HT7700 instrument at $120 \mathrm{kV}$. The static WCA of microfibrous membranes were measured using a contact angle analyzer (DSA25S, Data Physics Corporation).

\section{In vitro drug release tests.}

The test specimens $(20 \times 20 \mathrm{~mm}$, approximately $350 \mu \mathrm{m}$ thick; total mass $=50 \mathrm{mg})$ of PLA fibrous membranes were immersed in $10 \mathrm{~mL}$ phosphate buffered saline (PBS, $154 \mathrm{mM}$, pH 7.4). The suspension was placed in a $50 \mathrm{~mL}$ centrifugal tube and maintained in a thermostat shaker (Thermo, USA) at $37{ }^{\circ} \mathrm{C}$ with 100 cycles/min. Next, $2.0 \mathrm{~mL}$ release buffer was removed from the centrifugal tube and $2.0 \mathrm{~mL}$ fresh PBS was replaced at different time points. The quantity of released GEM from PLA fibrous membranes in release buffer was measured 


\section{WILEY-VCH}

by high-performance liquid chromatography (HPLC, Waters) with a mobile phase of $0.05 \mathrm{M}$ ammonium acetate buffer and methanol $(90: 10 \%)$ at a flow rate of $1 \mathrm{~mL} / \mathrm{min}$, a detection wavelength of $268 \mathrm{~nm}$, and a column $(\mathrm{C} 18,250 \mathrm{~mm} \times 4.6 \mathrm{~mm}$ for length, $5 \mu \mathrm{m}$ for filler size $)$ temperature of $30^{\circ} \mathrm{C}$. Based on the initial weight of GEM incorporated into the electrospun fibrous membrane, the percentage of released drug from the membranes was calculated.

\section{Cell culture and reagents.}

Human PDAC cell lines (PANC-1 and BxPC-3) and mouse fibroblast 3T3 cells were purchased from the Chinese Academy of Sciences. PANC-1 and 3T3 cells were cultured in Dulbecco's Modified Eagle's Medium (DMEM), and BxPC-3 cells were cultured in Roswell Park Memorial Institute-1640 (RPMI-1640) medium. All culture media contained 10\% fetal bovine serum (Gibco), $100 \mathrm{U} / \mathrm{mL}$ penicillin and $100 \mathrm{U} / \mathrm{mL}$ streptomycin (Sigma). Cells were cultured at $37{ }^{\circ} \mathrm{C}$ in a humidified atmosphere containing $5 \% \mathrm{CO}_{2}$.

\section{Phalloidin staining.}

Approximately $2 \times 10^{4}$ cells were seeded on PLA membranes in 48-well plates for 1 and 3 days. For fluorescence microscope observations, cells were washed with PBS, fixed in $4 \%$ formaldehyde solution for $30 \mathrm{~min}$ and washed twice with PBS. Subsequently, the cells were incubated with $0.1 \%$ Triton X-100 for 5 mins and phalloidin solution (Yearsen, China) for 30 min, washed twice with PBS, and finally incubated in 4', 6-diamidino-2-phenylindole (DAPI) solution for $10 \mathrm{~min}$ in the dark.

\section{Cell proliferation.}

Cell viability was evaluated with a cell counting kit (CCK-8, Dojindo, Japan). Fibrous materials were cut into the proper size to cover the wells in 24-well plates and placed into the bottom of the wells with an iron ring to prevent floating in the culture medium. All materials 


\section{WILEY-VCH}

were decontaminated under ultraviolet (UV) irradiation overnight. Approximately $4 \times 10^{4} 3 \mathrm{~T} 3$ or PANC-1 cells were seeded on the PLA electrospun membranes in 24-well plates for culturing for 1 and 3 days and cells cultured without membranes were used as the control. After incubation, the wells were refreshed with new culture medium with $10 \%$ CCK8 solution and cultured at $37^{\circ} \mathrm{C}$ for another $2 \mathrm{~h}$ to allow the reaction to proceed. Finally, $100 \mu \mathrm{L}$ reaction medium was transferred into 96-well plates for absorbance measurements at a wavelength of $450 \mathrm{~nm}$ with a microplate reader.

\section{Cell viability assay.}

The cytotoxicity of a GEM-loaded electrospun membrane against PANC-1 and BxPC-3 cells was assessed by a MTT assay (Yearsen, China). Approximately $4 \times 10^{4}$ PANC-1 and BxPC-3 cells were cultured in a 24 -well plate with culture medium overnight. Wells were divided into the following groups: (1) Control group, cells cultured in 24-well plates; (2) PLA control group, $5 \times 5$ mm PLA membranes were added in the bottom of the wells; (3) PLA-HA control group, 5×5 mm PLA-HA membranes were added; (4) GEM1@PLA-HA group, 5×5 mm GEM1@PLA-HA membrane were added; (5) GEM2@PLA-HA, 5×5 mm GEM2@PLA-HA membranes were added; and (6) GEM3@PLA-HA, 5×5 mm GEM3@PLA-HA membranes were added. All materials were decontaminated under UV irradiation overnight before the experiments. After culturing for 3, 5, and 7 days, MTT assays were performed for cell viability as measured by a microplate reader (MULTISKAN MK3, Thermo Scientific) at the wavelength of $490 \mathrm{~nm}$.

\section{Apoptosis assay.}

PANC-1 and BxPC-3 cell apoptosis induced by GEM-loaded PLA electrospun membranes was evaluated by flow cytometry (FCM) with Annexin V-fluorescein isothiocyanate (FITC)/PI double staining. Approximately $4 \times 10^{5}$ PANC-1 or BxPC-3 cells were seeded on 6- 


\section{WILEY-VCH}

well plates and cultured overnight, and GEM-loaded PLA electrospun membranes containing different amounts of GEM (GEM1@PLA-HA, GEM2@PLA-HA, and GEM3@PLA-HA) or PLA electrospun membranes (PLA-control) were placed into culture medium for 3 days of cell culture; all materials were cut into $1 \times 1 \mathrm{~cm}$ pieces and decontaminated under UV irradiation for $24 \mathrm{~h}$. Cells were collected, washed with cold PBS and centrifuged at $1500 \times g$ for 5 min. Next, the cells were incubated with Annexin V-FITC and PI solution (Yearsen, China) for 10 min and analyzed by FCM.

\section{Xenograft tumor model.}

Animal experiments were permitted by the Ethical Review Committee of the Sixth Affiliated Hospital of Shanghai Jiaotong University. Six-to-eight-week-old male BALB/c athymic mice (nude mice) were purchased from Shanghai Si Lai Ke Laboratory Animal Co., Ltd. China. Approximately $1 \times 10^{7}$ PANC-1 cells were subcutaneously inoculated into the right thigh of nude mice $(n=5)$. After the tumors grew to $70 \mathrm{~mm}^{3}$, the mice were divided into two groups. One group received peritoneal administration of GEM $(100 \mathrm{mg} / \mathrm{kg})$ and was denoted the GEM group. For the other group, a small incision was made on the skin to expose the tumor, and GEM3@PLA-HA membranes (cut as $2 \times 2 \mathrm{~cm}^{2}$ ) were placed on the tumor surface; this group was denoted the GEM3@PLA-HA group. Mice were weighed, and the tumor volume was calculated by caliper every 3 days as follows: $\mathrm{V}=0.5 \times\left(\right.$ length $\times$ width $\left.^{2}\right)$. After the mice were euthanized, tumors were collected for further studies; tumors were embedded in paraffin and cut into 5- $\mu \mathrm{m}$-thick sections for IHC analysis.

\section{Residual tumor model.}

Approximately $1 \times 10^{7}$ PANC-1 cells were subcutaneously inoculated into the right flank of nude mice $(n=5)$. After the tumor grew to $100 \mathrm{~mm}^{3}$, a small incision was made on the mouse skin to expose the tumor, and three-quarters of the tumor was removed (approximately $4 \mathrm{~mm}$ 


\section{WILEY-VCH}

$\times 4 \mathrm{~mm}$ of tumor tissue remained), as in our previous study ${ }^{26}$. Mice were divided into three groups, including the control, GEM and GEM3@PLA-HA groups. Mice in the control group were intraperitoneally injected with saline; Mice in the GEM3@PLA-HA group were locally implanted with GEM3@PLA-HA membrane; The mice in GEM group were intraperitoneally injected with GEM $(100 \mathrm{mg} / \mathrm{kg})$ twice a week. Before the materials were implanted, they were cut into $2 \times 2 \mathrm{~cm}$ in size. Mice were weighed, and tumor volume was calculated as follows: $\mathrm{V}=0.5 \times\left(\text { length } \times \text { width }^{2}\right)^{[36]}$. After the mice were euthanized, tumors and blood were collected for further analysis; tumors were embedded in paraffin and cut into 5- $\mu$ m-thick sections for IHC analysis. Plasma was harvested from blood by centrifugation at 3,000 rpm for 10 min. Next, liver function was tested by measuring AST and ALT.

\section{Immunohistochemical (IHC) staining analysis.}

Tumor tissues were fixed in $4 \%$ paraformaldehyde, embedded in paraffin, cut into in 5- $\mu$ mthick sections, deparaffinized with xylene, gradient-rehydrated with ethanol and subjected to antigen-retrieval with citrate buffer at $95{ }^{\circ} \mathrm{C}$ for $15 \mathrm{~min}$. Sections were later blocked with goat serum for 10 min and incubated with primary antibodies (Ki-67, caspase-3 and Bcl-2) for 30 min at room temperature. Next, the membrane was incubated with a goat anti-rabbit secondary antibody conjugated to horse radish peroxidase (HRP) for $10 \mathrm{~min}$ at $37{ }^{\circ} \mathrm{C}$. The targeted marker was detected with 3, 3'-diaminobenzidine (DAB) over 1-5 min. The nucleus was stained with hematoxylin. All staining was observed and photographed using a microscope (Nikon ECLIPSE 80i).

\section{IHC quantification.}

IHC staining for Bcl-2 was evaluated on the basis of staining intensity and staining proportion; at least five fields of $400 \times$ magnification were counted. The staining intensity was scored from grades 0 to 3 ( 0 , absent; 1, weak; 2, moderate; and 3, intense). The staining 


\section{WILEY-VCH}

proportion was scored from grades $1-3(1,<10 \% \text { of positive cells; } 2,10-49 \% ; 3,>50 \%)^{[27]}$. The IHC score was calculated by multiplying the scores of staining intensity by proportion. IHC staining for Ki-67 and cleaved caspase 3 was evaluated on the basis of the number of stained cells.

\section{Statistical analysis.}

Statistical Product and Service Solutions (SPSS) 18.0 software was applied for all statistical analysis. The results are presented as the mean \pm standard deviations (SD). For samples that were used for MTT analysis, an $\mathrm{n}=4$ or 5 was used, for samples that were used for in vivo experiment, an $\mathrm{n}=5$ was applicable. Multiple group comparisons were performed by applying one-way ANOVA, and post hoc Bonferroni was performed to test the differences. Two-group comparisons were performed by using Student's $t$-test. * p-value $<0.05$, ** p-value $<0.01$ and $* * *$ p-value $<0.001$ represented Statistically significant differences

\section{Acknowledgements}

G.X., H.Z., and R.C. contributed equally to this work. This work was supported in part by the Science and Technology Commission of Shanghai Municipality (18ZR1434200 and 18ZR1429100), Shanghai Municipal Education Commission-Gaofeng Clinical Medicine Grant Support (20171906), Excellent Youth Scholars of Shanghai Jiao Tong University School of Medicine (17XJ11004) and the State Key Laboratory of Molecular Engineering of Polymers (Fudan University K2018-02). Prof. Zhang acknowledges financial support from the Jane and Aatos Erkko Foundation (grant no. 4704010) and Academy of Finland (decision no. 297580). Prof. H. A. Santos acknowledges financial support from the University of Helsinki Research Funds, HiLIFE Research Funds, the Sigrid Jusélius Foundation (decision no. 4704580), and the European Research Council under the European Union's Seventh Framework Programme (FP/2007-2013, Grant No. 310892). 


\section{WILEY-VCH}

\section{Notes and references}

Received: ((will be filled in by the editorial staff))

Revised: ((will be filled in by the editorial staff)) Published online: ((will be filled in by the editorial staff))

[1] R. Siegel, J. Ma, Z. Zou, A. Jemal, CA Cancer J Clin 2014, 64, 9-29.

[2] M. Hidalgo, N Engl J Med 2010, 362, 1605-1617.

[3] a) T. Nordby, H. Hugenschmidt, M. W. Fagerland, T. Ikdahl, T. Buanes, K. J. Labori, Eur J Surg Oncol 2013, 39, 559-566; b) A. Van den Broeck, G. Sergeant, N. Ectors, W. Van Steenbergen, R. Aerts, B. Topal, Eur J Surg Oncol 2009, 35, 600-604.

[4] a) J. P. Neoptolemos, M. J. Moore, T. F. Cox, J. W. Valle, D. H. Palmer, A. C. McDonald, R. Carter, N. C. Tebbutt, C. Dervenis, D. Smith, B. Glimelius, R. M. Charnley, F. Lacaine, A. G. Scarfe, M. R. Middleton, A. Anthoney, P. Ghaneh, C. M. Halloran, M. M. Lerch, A. Olah, C. L. Rawcliffe, C. S. Verbeke, F. Campbell, M. W. Buchler, C. European Study Group for Pancreatic, JAMA 2012, 308, 147-156; b) H. Oettle, S. Post, P. Neuhaus, K. Gellert, J. Langrehr, K. Ridwelski, H. Schramm, J. Fahlke, C. Zuelke, C. Burkart, K. Gutberlet, E. Kettner, H. Schmalenberg, K. Weigang-Koehler, W. O. Bechstein, M. Niedergethmann, I. Schmidt-Wolf, L. Roll, B. Doerken, H. Riess, JAMA 2007, 297, 267277; c) J. P. Neoptolemos, D. D. Stocken, H. Friess, C. Bassi, J. A. Dunn, H. Hickey, H. Beger, L. Fernandez-Cruz, C. Dervenis, F. Lacaine, M. Falconi, P. Pederzoli, A. Pap, D. Spooner, D. J. Kerr, M. W. Buchler, C. European Study Group for Pancreatic, $N$ Engl J Med 2004, 350, 1200-1210.

[5] a) H. L. Kindler, T. Ioka, D. J. Richel, J. Bennouna, R. Letourneau, T. Okusaka, A. Funakoshi, J. Furuse, Y. S. Park, S. Ohkawa, G. M. Springett, H. S. Wasan, P. C. Trask, P. Bycott, A. D. Ricart, S. Kim, E. Van Cutsem, Lancet Oncol 2011, 12, 256-262; b) H. Maeda, G. Y. Bharate, J. Daruwalla, Eur J Pharm Biopharm 2009, 71, 409-419.

[6] a) D. Ohlund, A. Handly-Santana, G. Biffi, E. Elyada, A. S. Almeida, M. Ponz-Sarvise, V. Corbo, T. E. Oni, S. A. Hearn, E. J. Lee, Chio, II, C. I. Hwang, H. Tiriac, L. A. Baker, D. D. Engle, C. Feig, A. Kultti, M. Egeblad, D. T. Fearon, J. M. Crawford, H. Clevers, Y. Park, D. A. Tuveson, J Exp Med 2017, 214, 579-596; b) M. Erkan, S. Hausmann, C. W. Michalski, A. A. Fingerle, M. Dobritz, J. Kleeff, H. Friess, Nat Rev Gastroenterol Hepatol 2012, 9, 454-467; c) D. Mahadevan, D. D. Von Hoff, Mol Cancer Ther 2007, 6, 1186-1197.

[7] K. Uesaka, N. Boku, A. Fukutomi, Y. Okamura, M. Konishi, I. Matsumoto, Y. Kaneoka, 


\section{WILEY-VCH}

Y. Shimizu, S. Nakamori, H. Sakamoto, S. Morinaga, O. Kainuma, K. Imai, N. Sata, S.

Hishinuma, H. Ojima, R. Yamaguchi, S. Hirano, T. Sudo, Y. Ohashi, J. S. Group, Lancet 2016, $388,248-257$.

[8] H. Ueno, T. Kosuge, Y. Matsuyama, J. Yamamoto, A. Nakao, S. Egawa, R. Doi, M. Monden, T. Hatori, M. Tanaka, M. Shimada, K. Kanemitsu, Br J Cancer 2009, 101, 908915.

[9] B. B. Cerqueira, A. Lasham, A. N. Shelling, R. Al-Kassas, Eur J Pharm Biopharm 2015, 97, 140-151.

[10] a) A. Bahrami, M. Khazaei, F. Bagherieh, M. Ghayour-Mobarhan, M. Maftouh, S. M. Hassanian, A. Avan, J Cell Physiol 2017; b) P. P. Provenzano, C. Cuevas, A. E. Chang, V. K. Goel, D. D. Von Hoff, S. R. Hingorani, Cancer Cell 2012, 21, 418-429; c) K. P. Olive, M. A. Jacobetz, C. J. Davidson, A. Gopinathan, D. McIntyre, D. Honess, B. Madhu, M. A. Goldgraben, M. E. Caldwell, D. Allard, K. K. Frese, G. Denicola, C. Feig, C. Combs, S. P. Winter, H. Ireland-Zecchini, S. Reichelt, W. J. Howat, A. Chang, M. Dhara, L. Wang, F. Ruckert, R. Grutzmann, C. Pilarsky, K. Izeradjene, S. R. Hingorani, P. Huang, S. E. Davies, W. Plunkett, M. Egorin, R. H. Hruban, N. Whitebread, K. McGovern, J. Adams, C. Iacobuzio-Donahue, J. Griffiths, D. A. Tuveson, Science 2009, 324, 14571461.

[11] a) J. B. Wolinsky, Y. L. Colson, M. W. Grinstaff, J Control Release 2012, 159, 14-26; b) Y. Wang, P. Li, T. Truong-Dinh Tran, J. Zhang, L. Kong, Nanomaterials (Basel) 2016, 6; c) X. Z. Wenguo Cui, Yuguang Zhang, Plast Aesthet Res 2016, 3, 70-71; dV. S. Zhi Yuan(William) Lin, Arvind Dhinakar, Lara Yildirimer, Wenguo Cui, Xin Zhao, Plast Aesthet Res 2016, 3, 72-82.

[12] a) N. Goonoo, A. Bhaw-Luximon, D. Jhurry, J Biomed Nanotechnol 2014, 10, $2173-$ 2199; b) S. Zong, X. Wang, Y. Yang, W. Wu, H. Li, Y. Ma, W. Lin, T. Sun, Y. Huang, Z. Xie, Y. Yue, S. Liu, X. Jing, Eur J Pharm Biopharm 2015, 93, 127-135.

[13] a) C. Y. Ang, S. Y. Tan, C. Teh, J. M. Lee, M. F. Wong, Q. Qu, L. Q. Poh, M. Li, Y. Zhang, V. Korzh, Y. Zhao, Small 2017, 13; b) X. Zhao, Z. Yuan, L. Yildirimer, J. Zhao, Z. Y. Lin, Z. Cao, G. Pan, W. Cui, Small 2015, 11, 4284-4291; c) Z. Shi, X. Gao, M. W. Ullah, S. Li, Q. Wang, G. Yang, Biomaterials 2016, 111, 40-54. [14]L. Zhou, C. Zhu, W. Cui, H. Yang, B. Li, J Control Release 2015, 213, e10. [15] a) Z. Chen, Z. Chen, A. Zhang, J. Hu, X. Wang, Z. Yang, Biomater Sci 2016, 4, 922-932;

b) S. F. Chou, D. Carson, K. A. Woodrow, J Control Release 2015, 220, 584-591. [16] Y. Zhu, C. Hu, B. Li, H. Yang, Y. Cheng, W. Cui, Acta Biomater 2013, 9, 8328-8336. 


\section{WILEY-VCH}

[17]P. McClellan, W. J. Landis, Biores Open Access 2016, 5, 212-227.

[18]X. Sun., L. Cheng., J. Zhao., R. Jin., B. Sun., Y. Shi., L. Zhang., Y. Zhang*., W. Cui*. J. Mater. Chem. B 2014, 2, 3636-3645.

[19] Y. Lu, J. Huang, G. Yu, R. Cardenas, S. Wei, E. K. Wujcik, Z. Guo, Wiley Interdiscip Rev Nanomed Nanobiotechnol 2016, 8, 654-677.

[20] Y. Z. Zhang, X. Wang, Y. Feng, J. Li, C. T. Lim, S. Ramakrishna, Biomacromolecules 2006, 7, 1049-1057.

[21] N. Han, J. Johnson, J. J. Lannutti, J. O. Winter, J Control Release 2012, 158, 165-170.

[22] W. Cui, X. Li, X. Zhu, G. Yu, S. Zhou, J. Weng, Biomacromolecules 2006, 7, 1623-1629.

[23]P. Wen., K. Feng., H. Yang., X. Huang., M.-H. Zong., W.-Y. Lou., N. Li., H. Wu., Carbohydr Polym 2017, 169, 157-166.

[24] a) R. Kalluri, M. Zeisberg, Nat Rev Cancer 2006, 6, 392-401; b) B. C. Ozdemir, T. Pentcheva-Hoang, J. L. Carstens, X. Zheng, C. C. Wu, T. R. Simpson, H. Laklai, H. Sugimoto, C. Kahlert, S. V. Novitskiy, A. De Jesus-Acosta, P. Sharma, P. Heidari, U. Mahmood, L. Chin, H. L. Moses, V. M. Weaver, A. Maitra, J. P. Allison, V. S. LeBleu, R. Kalluri, Cancer Cell 2014, 25, 719-734.

[25] N. M. Aiello, T. Brabletz, Y. Kang, M. A. Nieto, R. A. Weinberg, B. Z. Stanger, Nature 2017, 547, E7-E8.

[26]Q. Xia, Z. Liu, C. Wang, Z. Zhang, S. Xu, C. C. Han, Biomacromolecules 2015, 16, 3083-3092.

[27]F. Vena, E. Li Causi, M. Rodriguez-Justo, S. Goodstal, T. Hagemann, J. A. Hartley, D. Hochhauser, Clin Cancer Res 2015, 21, 5563-5577.

[28]D. D. Von Hoff, T. Ervin, F. P. Arena, E. G. Chiorean, J. Infante, M. Moore, T. Seay, S. A. Tjulandin, W. W. Ma, M. N. Saleh, M. Harris, M. Reni, S. Dowden, D. Laheru, N. Bahary, R. K. Ramanathan, J. Tabernero, M. Hidalgo, D. Goldstein, E. Van Cutsem, X. Wei, J. Iglesias, M. F. Renschler, N Engl J Med 2013, 369, 1691-1703.

[29] a) I. T. Konstantinidis, A. L. Warshaw, J. N. Allen, L. S. Blaszkowsky, C. F. Castillo, V. Deshpande, T. S. Hong, E. L. Kwak, G. Y. Lauwers, D. P. Ryan, J. A. Wargo, K. D. Lillemoe, C. R. Ferrone, Ann Surg 2013, 257, 731-736; b) M. Bockhorn, F. G. Uzunoglu, M. Adham, C. Imrie, M. Milicevic, A. A. Sandberg, H. J. Asbun, C. Bassi, M. Buchler, R. M. Charnley, K. Conlon, L. F. Cruz, C. Dervenis, A. Fingerhutt, H. Friess, D. J. Gouma, W. Hartwig, K. D. Lillemoe, M. Montorsi, J. P. Neoptolemos, S. V. Shrikhande, K. Takaori, W. Traverso, Y. K. Vashist, C. Vollmer, C. J. Yeo, J. R. Izbicki, S. International Study Group of Pancreatic, Surgery 2014, 155, 977-988. 


\section{WILEY-VCH}

[30]H. A. Burris, 3rd, M. J. Moore, J. Andersen, M. R. Green, M. L. Rothenberg, M. R. Modiano, M. C. Cripps, R. K. Portenoy, A. M. Storniolo, P. Tarassoff, R. Nelson, F. A. Dorr, C. D. Stephens, D. D. Von Hoff, J Clin Oncol 1997, 15, 2403-2413.

[31] a) M. A. Jacobetz, D. S. Chan, A. Neesse, T. E. Bapiro, N. Cook, K. K. Frese, C. Feig, T. Nakagawa, M. E. Caldwell, H. I. Zecchini, M. P. Lolkema, P. Jiang, A. Kultti, C. B. Thompson, D. C. Maneval, D. I. Jodrell, G. I. Frost, H. M. Shepard, J. N. Skepper, D. A. Tuveson, Gut 2013, 62, 112-120; b) M. H. Sherman, R. T. Yu, D. D. Engle, N. Ding, A. R. Atkins, H. Tiriac, E. A. Collisson, F. Connor, T. Van Dyke, S. Kozlov, P. Martin, T. W. Tseng, D. W. Dawson, T. R. Donahue, A. Masamune, T. Shimosegawa, M. V. Apte, J. S. Wilson, B. Ng, S. L. Lau, J. E. Gunton, G. M. Wahl, T. Hunter, J. A. Drebin, P. J. O'Dwyer, C. Liddle, D. A. Tuveson, M. Downes, R. M. Evans, Cell 2014, 159, 80-93.

[32] S. T. Yohe, V. L. Herrera, Y. L. Colson, M. W. Grinstaff, J Control Release 2012, 162, 92101.

[33] a) C. P. Zambirinis, G. Miller, Trends Mol Med 2017; b) A. Neesse, P. Michl, K. K. Frese, C. Feig, N. Cook, M. A. Jacobetz, M. P. Lolkema, M. Buchholz, K. P. Olive, T. M. Gress, D. A. Tuveson, Gut 2011, 60, 861-868; c) R. Kadaba, H. Birke, J. Wang, S. Hooper, C. D. Andl, F. Di Maggio, E. Soylu, M. Ghallab, D. Bor, F. E. Froeling, S. Bhattacharya, A. K. Rustgi, E. Sahai, C. Chelala, P. Sasieni, H. M. Kocher, J Pathol 2013, 230, 107-117.

[34]R. Langer, Science 1990, 249, 1527-1533.

[35] V. Heinemann, M. Reni, M. Ychou, D. J. Richel, T. Macarulla, M. Ducreux, Cancer Treat Rev 2014, 40, 118-128.

[36] R. Chugh, V. Sangwan, S. P. Patil, V. Dudeja, R. K. Dawra, S. Banerjee, R. J. Schumacher, B. R. Blazar, G. I. Georg, S. M. Vickers, A. K. Saluja, Sci Transl Med 2012, 4, 156 ra139. 


\section{WILEY-VCH}

\section{Figure Captions}

Figure 1. (A) Schematic illustration of the process of constructing GEM-loaded PLA electrospun fibrous membranes withsol-electrospinning technology. (B) SEM images of PLA (a), GEM1@PLA-HA (b), GEM2@PLA-HA (c), GEM3@PLA-HA (d), GEM1@PLA-HA2 (e), and GEM1@PLA-HA3 (f) fibers (insert pictures are TEM images of the fiber to show the core-shell). (C) GEM release profiles of fibers containing different drug content (GEM1@PLA-HA, GEM2@PLA-HA and GME3@PLA-HA) and different sol-content (GEM1@PLA-HA, GEM1@PLA-HA2 and GEM1@PLA-HA3) $(n=3)$. Data represent the mean \pm SD.

Figure 2. (A) Cell viability of $3 \mathrm{~T} 3$ and PANC-1 cells grown on PLA or PLA-HA eletrospun membranes for 1 and 3 days $(n=4)$. (B) Morphology of 3T3 and PANC- 1 cells grown on PLA or PLA-HA electrospun membranes or culture plates for 1 and 3 days of incubation. Red scale bars indicate $100 \mu \mathrm{m}$. (C) Morphology of 3T3 and PANC-1 cells grown on PLA or PLA-HA electrospun membranes for 3 days were imaged by SEM. Scale bars indicate $50 \mu \mathrm{m}$. Data represent the mean $\pm \mathrm{SD}$, and $* *$ indicates $p<0.01$.

Figure 3. (A) Cell viability of PANC-1 and BxPC-3 cells treated with GEM-loaded electrospun membranes (PLA, PLA-HA, GEM1@PLA-HA, and GEM2@PLA-HA, GEM3@PLA-HA) for 3, 5, 7 days $(n=5)$. (B) Flow cytometry of AnnexinV/PI double staining: cell apoptosis of PANC-1 and BxPC-3 cells treated with GEM-loaded electrospun membranes (PLA, PLA-HA, GEM1@PLA-HA, GEM2@PLA-HA, and GEM3@PLA-HA) for 3 days. Data represent the mean $\pm \operatorname{SD}(n=5)$. * indicates $p<0.05$ and $* * *$ indicates $p<$ 0.001 . 


\section{WILEY-VCH}

Figure 4. (A) Schematic illustration of implantation of GEM3@PLA-HA on the tumor of a xenograft tumor model of pancreatic cancer for local chemotherapy. (B) Xenograft tumor tissues of pancreatic cancer $(n=5)$. (C) Tumor volume of the GEM3@PLA-HA and GEM groups. (D) Tumor weight of the GEM3@PLA-HA and GEM groups. (E) Mouse weight of the GEM3@PLA-HA and GEM groups. (F) IHC staining of Bcl-2, Cleaved-caspase-3 and Ki-67. Black scale bars indicate $200 \mu \mathrm{m}$. (G) Semi-quantification of Bcl-2, Cleaved-caspase-3 and Ki-67. Data represent the mean \pm SD. * indicates $P<0.05$.

Figure 5. (A) Schematic illustration of implantation of GEM3@PLA-HA on the residual tumor of a xenograft residual tumormodel for local chemotherapy. (B) Xenograft residual tumor of pancreatic cancer $(n=5)$. (C) Tumor weight of the control, GEM and GEM3@PLAHAGEM groups. (D) Tumor volume of the control, GEM and GEM3@PLA-HAGEM groups. (E) Mice weight of the control, GEM and GEM3@PLA-HAGEM groups. (F) IHC staining of Bcl-2, Cleaved-caspase-3 and Ki-67. Black scale bars indicate $200 \mu \mathrm{m}$. (G) Semiquantification of Bcl-2, Cleaved-caspase-3 and Ki-67. Data represents mean \pm SD. *** indicates $P<0.001$, ** indicates $P<0.01$.

Figure 6. (A) HE staining of the liver after treatment with GEM3@PLA-HA and GEM. Black arrows showed the necrosis of liver cells. Black scale bars indicate $200 \mu \mathrm{m}$. (B) AST and ALT levels of mice treated with GEM3@PLA-HA or GEM. The control group included normal nude mice without tumors $(n=5)$. Data represent the mean \pm SD. ** indicates $P<$ 0.01 and $* * *$ indicates $P<0.001$.

Figure 7. Schematic illustration of the GEM-loaded PLA eletrospun membrane on pancreatic cancer for local chemotherapy. (A) Before surgery, tumor integrity is maintained, and the fibrous tissue around the tumor could prevents GEM from penetrating tumor tissues. (B) After 


\section{WILEY-VCH}

surgery, when tumor is incompeletly resected, tumor integrity is destroyed, and the fibrous tissue around the tumor is also destroyed, therefore GEM released from the GEM-loaded PLA electrospun fibrous membranes can easily penetrate tumor tissues. (C) After surgery, when tumor is compeletly resected, and GEM released from the GEM-loaded PLA electrospun fibrous membranes can prevent the local tumor recurrence by killing the pancreatic cancer cells which may be existed in the surgical site. 


\section{WILEY-VCH}

\section{Figures}

(A)

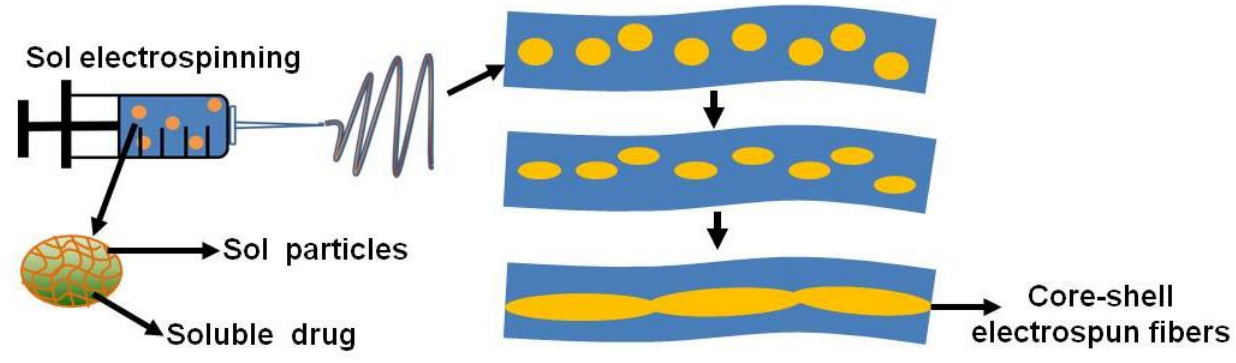

(B)
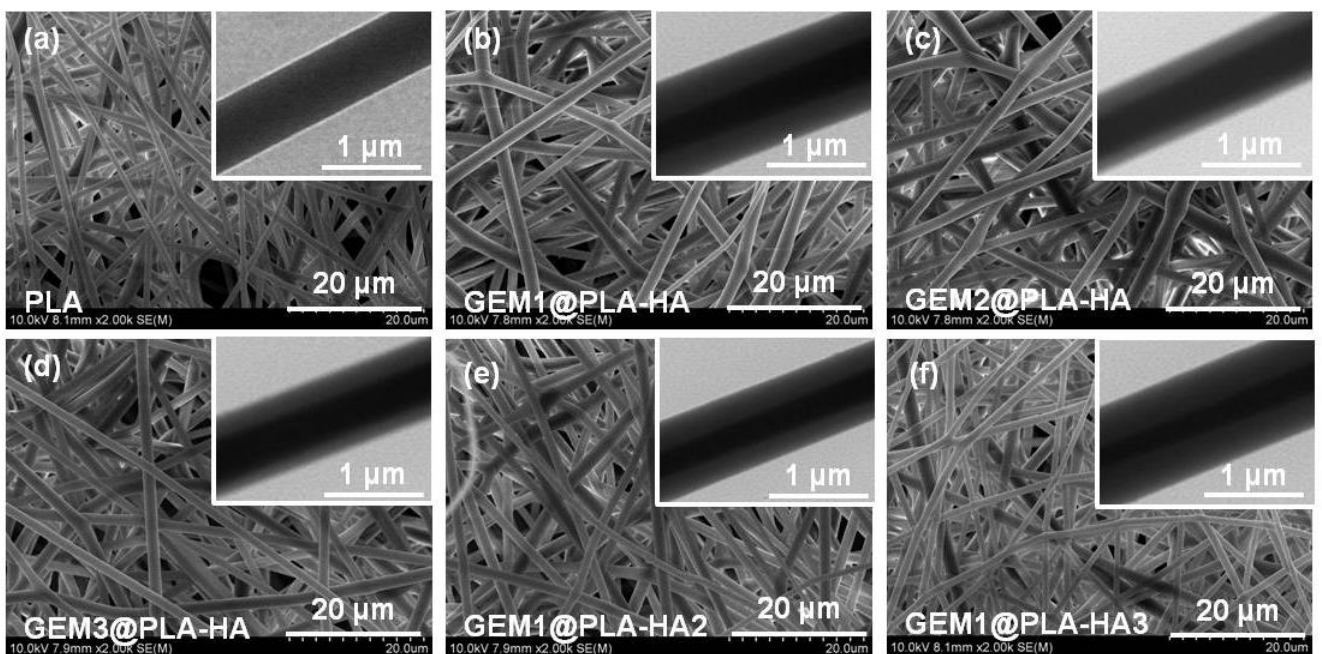

(C)
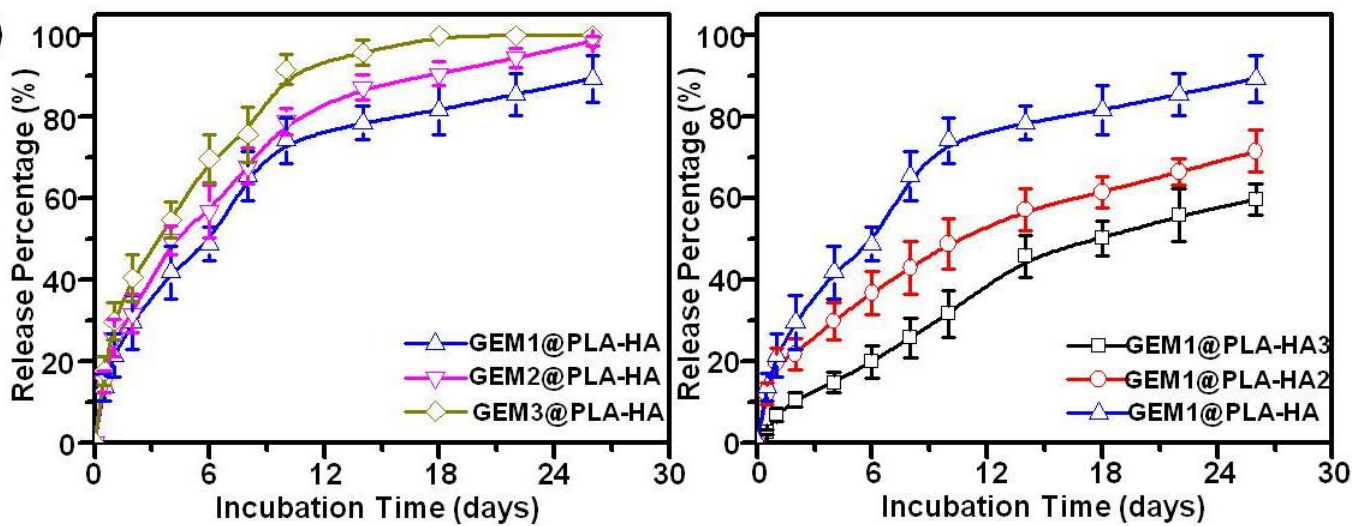

Figure 1. (A) Schematic illustration of the process of constructing GEM-loaded PLA electrospun fibrous membranes with sol-electrospinning technology. (B) SEM images of PLA (a), GEM1@PLA-HA (b), GEM2@PLA-HA (c), GEM3@PLA-HA (d), GEM1@PLA-HA2 (e), and GEM1@PLA-HA3 (f) fibers (insert pictures are TEM images of the fiber to show the core-shell). (C) GEM release profiles of fibers containing different drug content (GEM1@PLA-HA, GEM2@PLA-HA and GME3@PLA-HA) and different sol-content 
WILEY-VCH

(GEM1@PLA-HA, GEM1@PLA-HA2 and GEM1@PLA-HA3) $(n=3)$. Data represent the mean \pm SD. 


\section{WILEY-VCH}

(A)
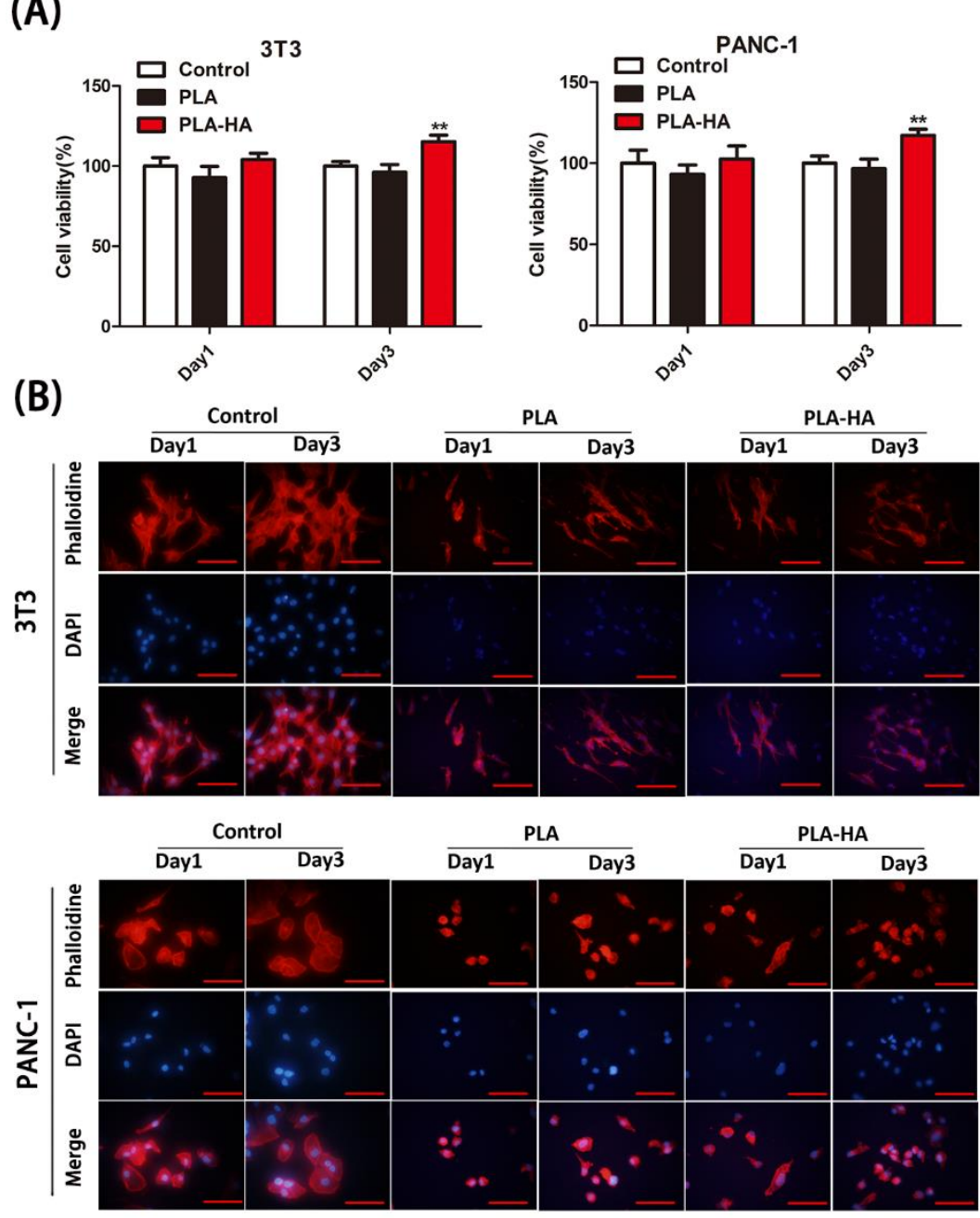

(C)

3T3

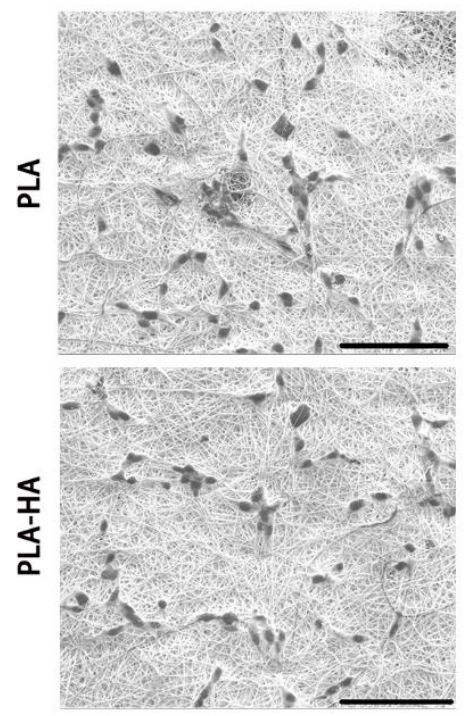

PANC-1

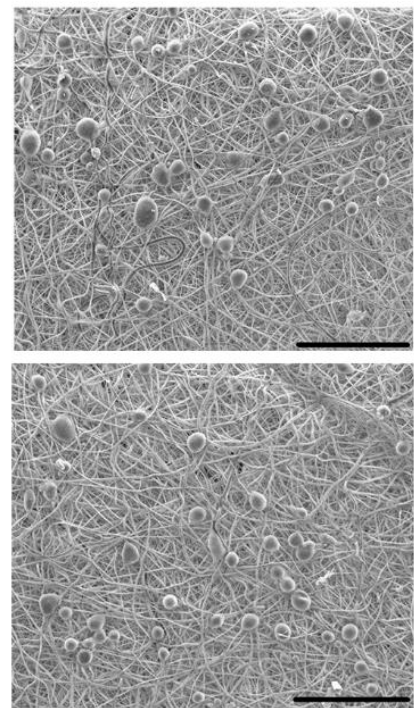

Figure 2. (A) Cell viability of 3T3 and PANC-1 cells grown on PLA or PLA-HA eletrospun membranes for 1 and 3 days $(n=4)(B)$ Morphology of 3 T3 and PANC-1 cells grown on PLA or PLA-HA electrospun membranes or culture plates for 1 and 3 days of incubation. Red scale 


\section{WILEY-VCH}

bars indicate $100 \mu \mathrm{m}$. (C) Morphology of 3T3 and PANC-1 cells grown on PLA or PLA-HA electrospun membranes for 3 days were imaged by SEM. Scale bars indicate $50 \mu \mathrm{m}$. Data represent the mean $\pm \mathrm{SD}, * *$ indicates $p<0.01$. 


\section{WILEY-VCH}

(A)
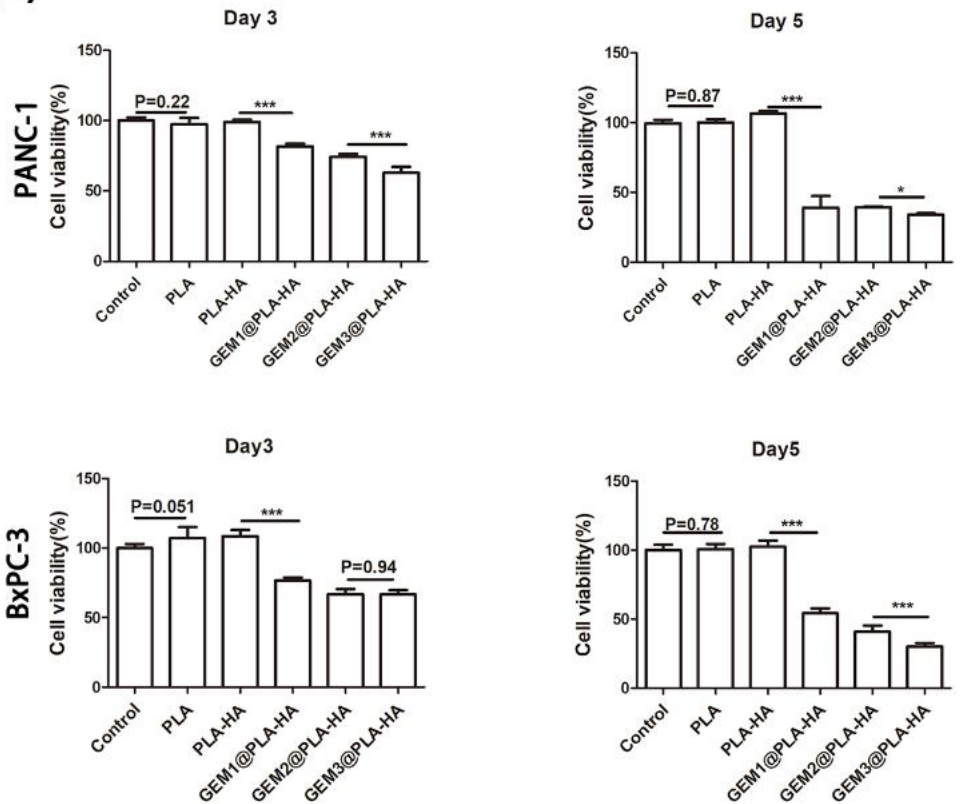

(B)

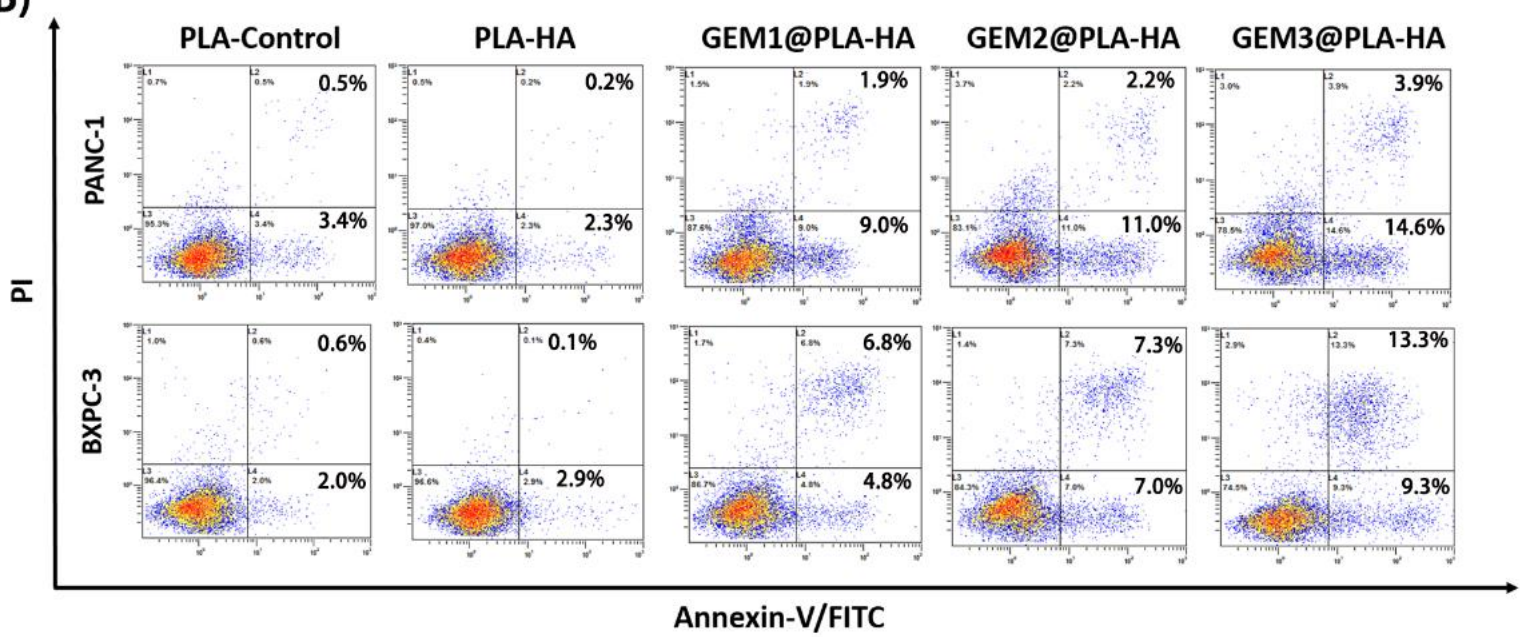

Figure 3. (A) Cell viability of PANC-1 and BxPC-3 cells treated with GEM-loaded electrospun membranes (PLA, PLA-HA, GEM1@PLA-HA, and GEM2@PLA-HA, GEM3@PLA-HA) for 3, 5, 7 days $(n=5)$. (B) Flow cytometry of AnnexinV/PI double staining: cell apoptosis of PANC-1 and BxPC-3 cells treated with GEM-loaded electrospun membranes (PLA, PLA-HA, GEM1@PLA-HA, GEM2@PLA-HA, and GEM3@PLA-HA) for 3 days. Data represent the mean \pm SD. $*$ indicates $p<0.05$ and $* * *$ indicates $p<0.001$. 


\section{WILEY-VCH}

(A)

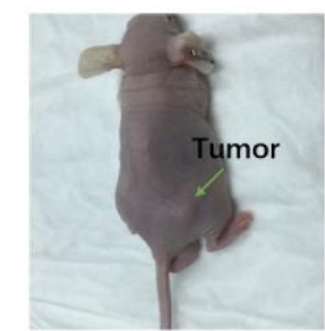

(C)

(F)

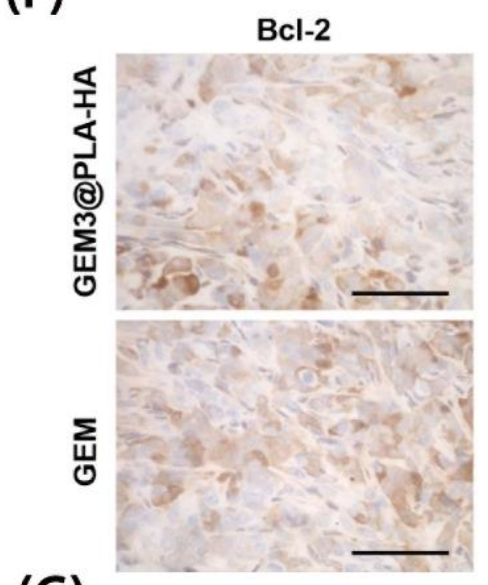

(G)

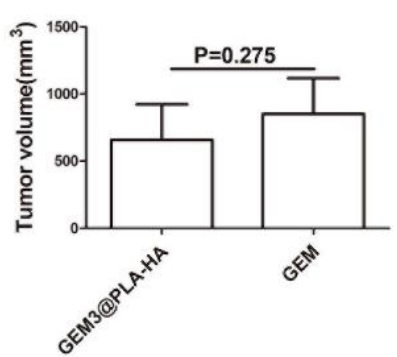

(D)

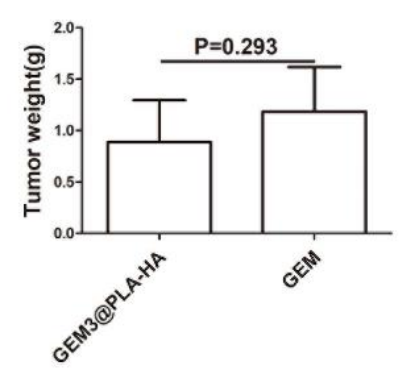

Cleaved-caspase 3

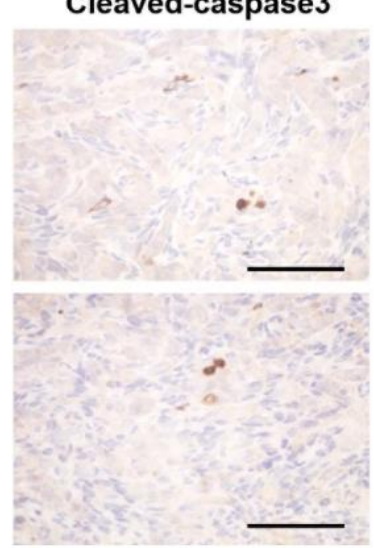

Cleaved-caspase 3

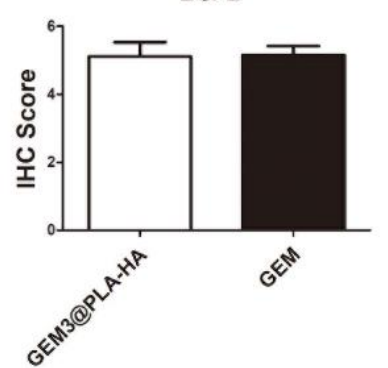

(B)
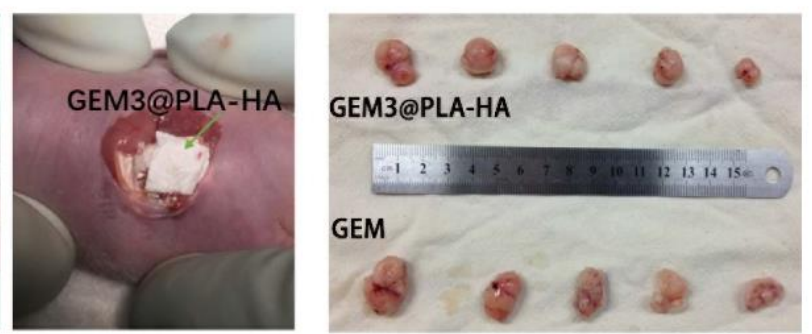

$(E)^{26}=$ GEM3@PLA-HA

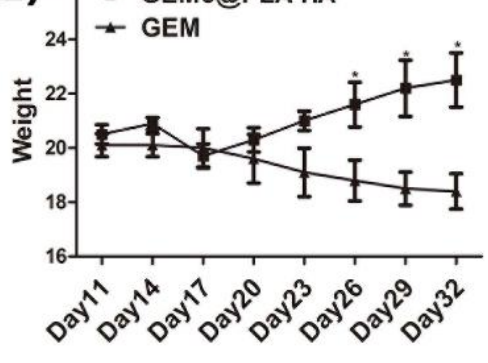

Ki-67

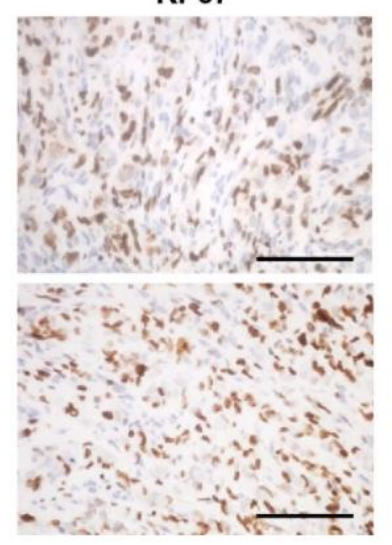

Ki-67

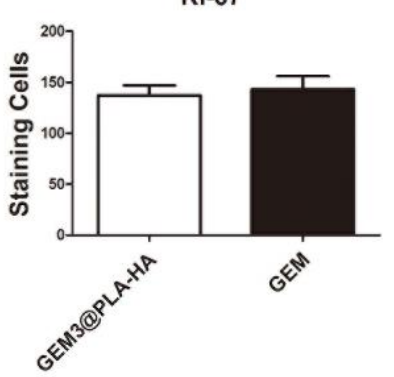

Figure 4. (A) Schematic illustration of implantation of GEM3@PLA-HA on the tumor of a xenograft tumor model of pancreatic cancer for local chemotherapy. (B) Xenograft tumor tissues of pancreatic cancer $(n=5)$. (C) Tumor volume of the GEM3@PLA-HA and GEM groups. (D) Tumor weight of the GEM3@PLA-HA and GEM groups. (E) Mice weight of the GEM3@PLA-HA and GEM groups. Black scale bars indicate $200 \mu \mathrm{m}$. (F) IHC staining of 


\section{WILEY-VCH}

Bcl-2, Cleaved-caspase-3 and Ki-67. (G) Semi-quantification of Bcl-2, Cleaved-caspase-3 and Ki-67. Data represent the mean \pm SD. * indicates $P<0.05$. 
(A)

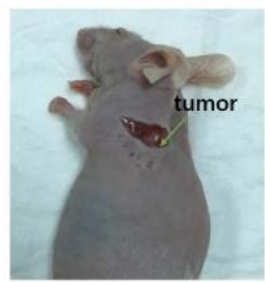

(C)

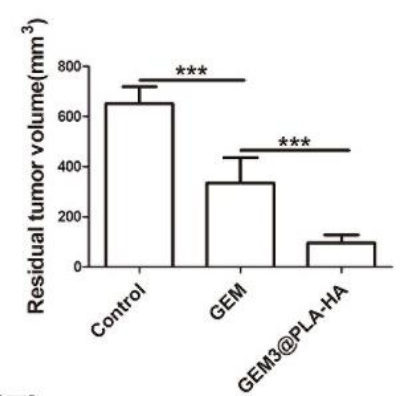

(F)

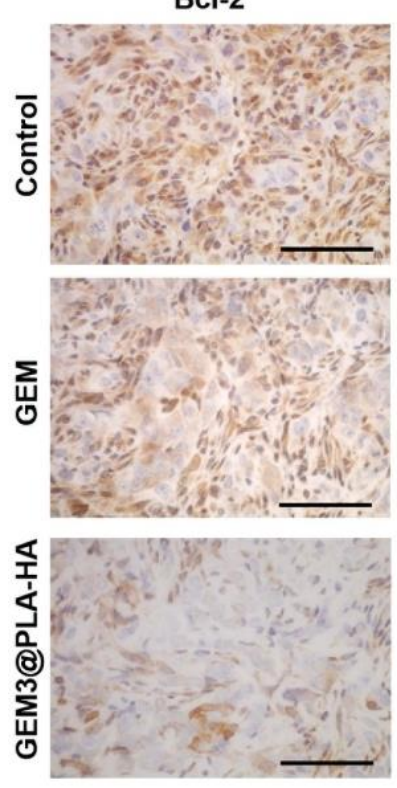

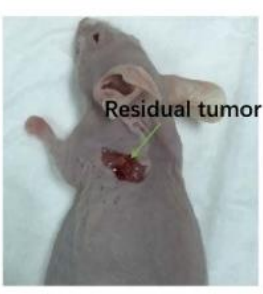

(D)

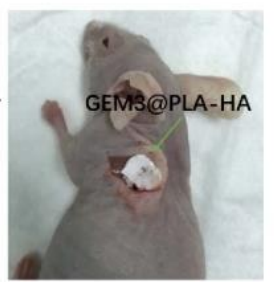

(E)

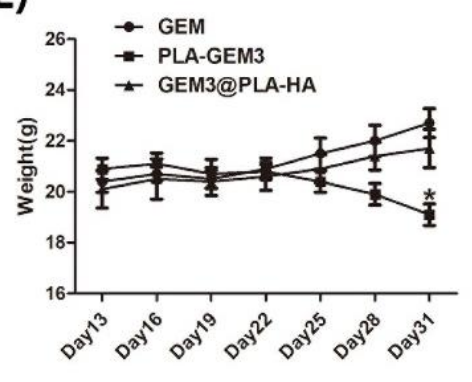

(G)

(B)
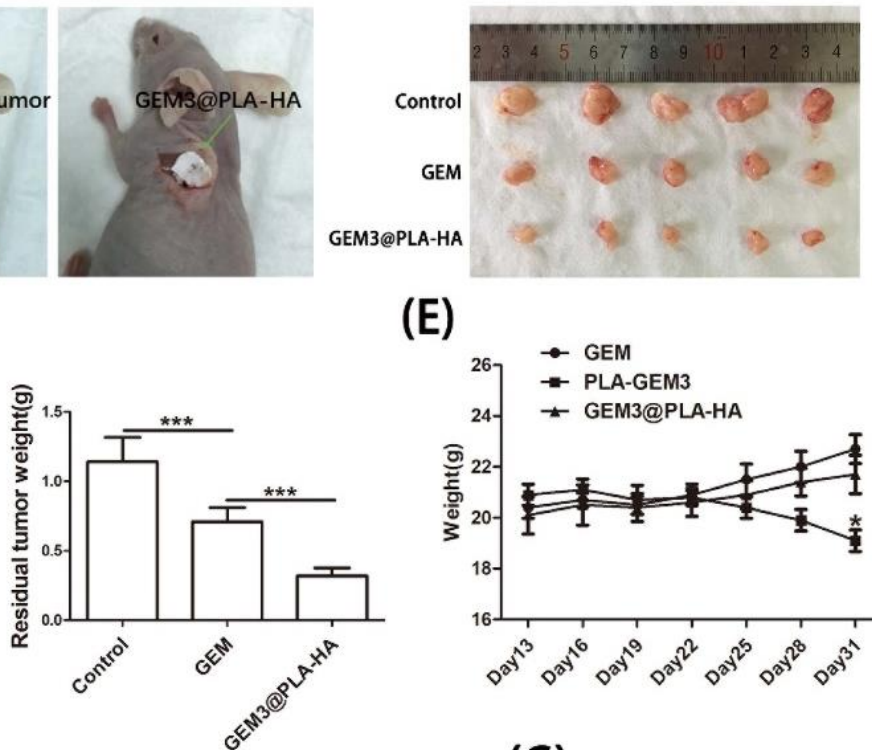

Ki-67
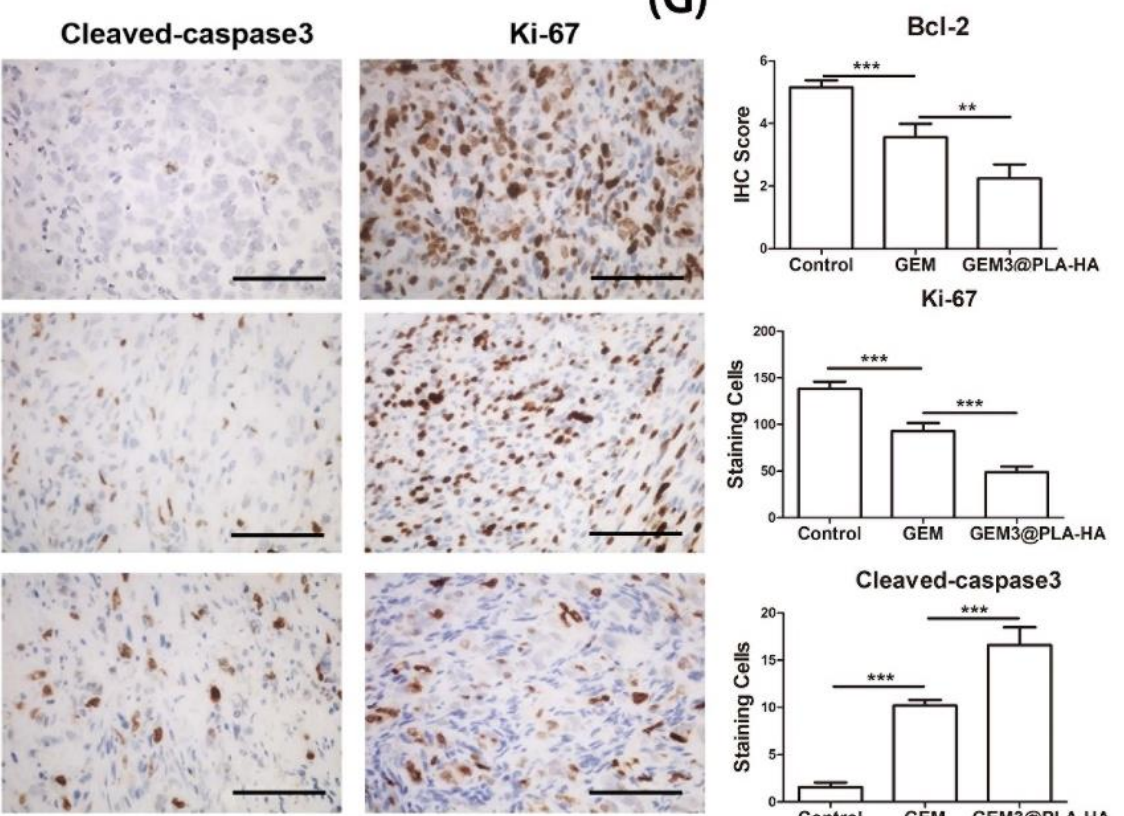

Figure 5. (A) Schematic illustration of implantation of GEM3@PLA-HA on the residual tumor of a xenograft residual tumor model for local chemotherapy. (B) Xenograft residual tumor of pancreatic cancer $(n=5)$. (C) Tumor weight of the control, GEM and GEM3@PLAHAGEM groups. (D) Tumor volume of the control, GEM and GEM3@PLA-HAGEM groups. (E) Mice weight of the control, GEM and GEM3@PLA-HAGEM groups. (F) IHC staining of Bcl-2, Cleaved-caspase-3 and Ki-67. Black scale bars indicate $200 \mu \mathrm{m}$. (G) Semi- 
WILEY-VCH

quantification of Bcl-2, Cleaved-caspase-3 and Ki-67. Data represent the mean \pm SD. *** indicates $P<0.001$, ** indicates $P<0.01$. 


\section{WILEY-VCH}

(A)
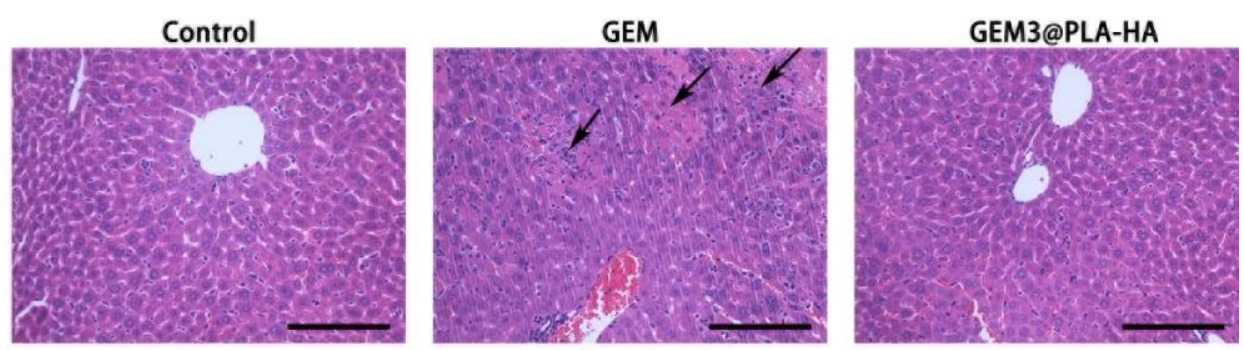

(B)
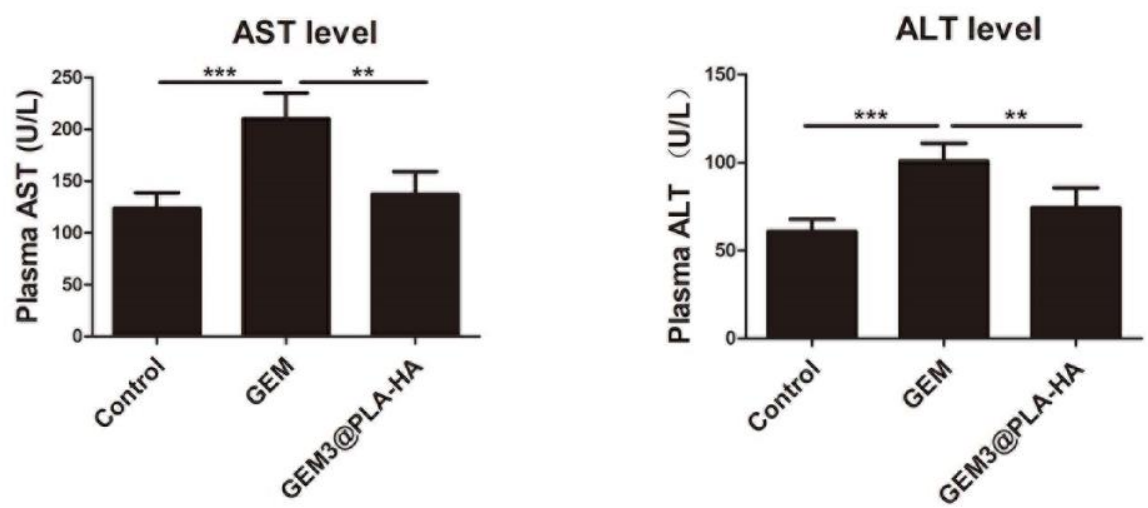

Figure 6. (A) HE staining of the liver after treatment with GEM3@PLA-HA and GEM. Black arrows showed the necrosis of liver cells. Black scale bars indicate $200 \mu \mathrm{m}$. (B) AST and ALT levels of mice treated with GEM3@PLA-HA or GEM. The control group included normal nude mice without tumors $(n=5)$. Data represent the mean \pm SD. ** indicates $P<$ 0.01 and $* * *$ indicates $P<0.001$. 


\section{WILEY-VCH}

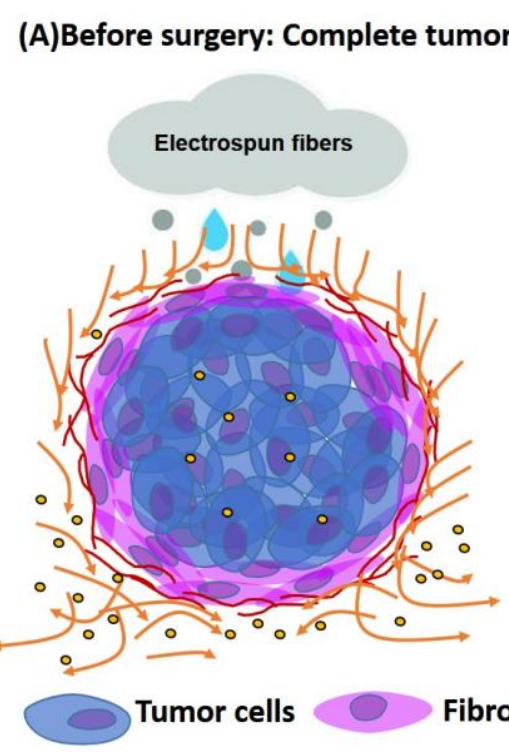

(B) After surgery: Resected tumor

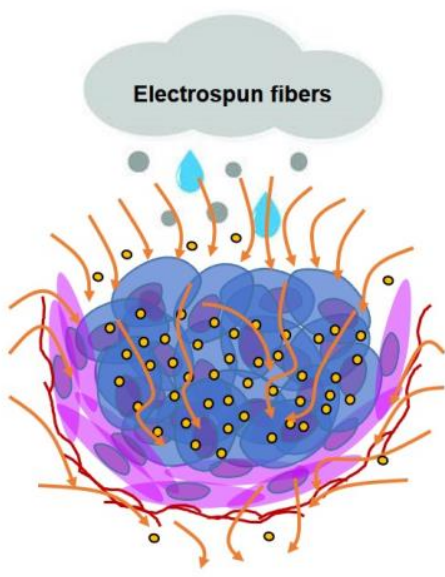

Fibrous membrane $\bigcirc$ Gemcitabine

(C) After surgery: Resected tumor

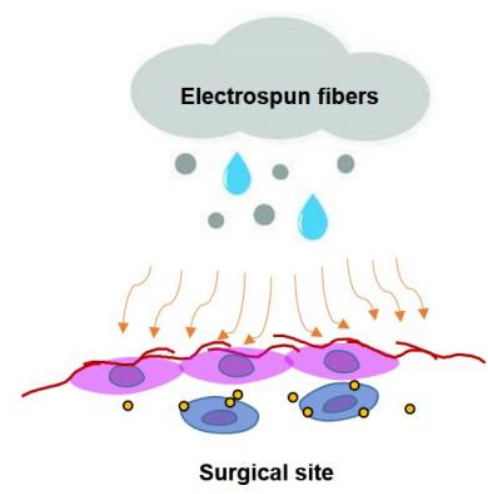

Figure 7. Schematic illustration of the GEM-loaded PLA eletrospun membrane on pancreatic cancer for local chemotherapy. (A) Before surgery, tumor integrity is maintained, and the fibrous tissue around the tumor could prevents GEM from penetrating tumor tissues. (B) After surgery, when tumor is incompeletly resected, tumor integrity is destroyed, and the fibrous tissue around the tumor is also destroyed, therefore GEM released from the GEM-loaded PLA electrospun fibrous membranes can easily penetrate tumor tissues. (C) After surgery, when tumor is completely resected, and GEM released from the GEM-loaded PLA electrospun fibrous membranes can prevent the local tumor recurrence by killing the pancreatic cancer cells which may be existed in the surgical site. 


\section{WILEY-VCH}

\section{TABLES}

Table 1. The components content of electrospun fibers groups.

\begin{tabular}{|c|c|c|c|c|c|}
\hline Sample Name & $\begin{array}{l}\text { PLA } \\
(\mathrm{g})\end{array}$ & $\begin{array}{c}\text { HA sol } \\
(\mu \mathrm{L})\end{array}$ & $\begin{array}{l}\text { GEM } \\
(\mathrm{mg})\end{array}$ & $\begin{array}{l}\text { Fiber Diameter } \\
\qquad(\mu \mathrm{m})\end{array}$ & $\begin{array}{c}\text { Core Diameter } \\
\qquad(\mu \mathrm{m})\end{array}$ \\
\hline PLA & 1 & 0 & 0 & $1.15 \pm 0.38$ & - \\
\hline GEM1@PLA-HA & 1 & 400 & 10 & $1.26 \pm 0.35$ & $0.37 \pm 0.07$ \\
\hline GEM2@PLA-HA & 1 & 400 & 15 & $1.32 \pm 0.46$ & $0.48 \pm 0.11$ \\
\hline GEM3@PLA-HA & 1 & 400 & 20 & $1.41 \pm 0.33$ & $0.39 \pm 0.09$ \\
\hline $\begin{array}{c}\text { GEM1@PLA- } \\
\text { HA2 }\end{array}$ & 1 & 200 & 10 & $1.30 \pm 0.47$ & $0.30 \pm 0.08$ \\
\hline $\begin{array}{c}\text { GEM1@PLA- } \\
\text { HA3 }\end{array}$ & 1 & 100 & 10 & $1.21 \pm 0.39$ & $0.24 \pm 0.06$ \\
\hline
\end{tabular}




\section{WILEY-VCH}
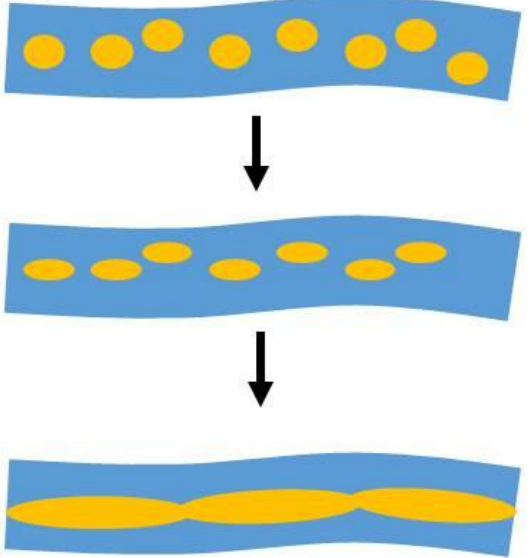

Core-shell electrospun fibers

\section{Graphical Abstract}


Click here to access/download Supporting Information

\section{Table of Content.docx}


Fig. 1

\title{
Click here to access/download \\ Production Data Figure 1.tif

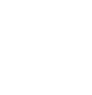

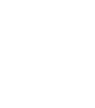

if
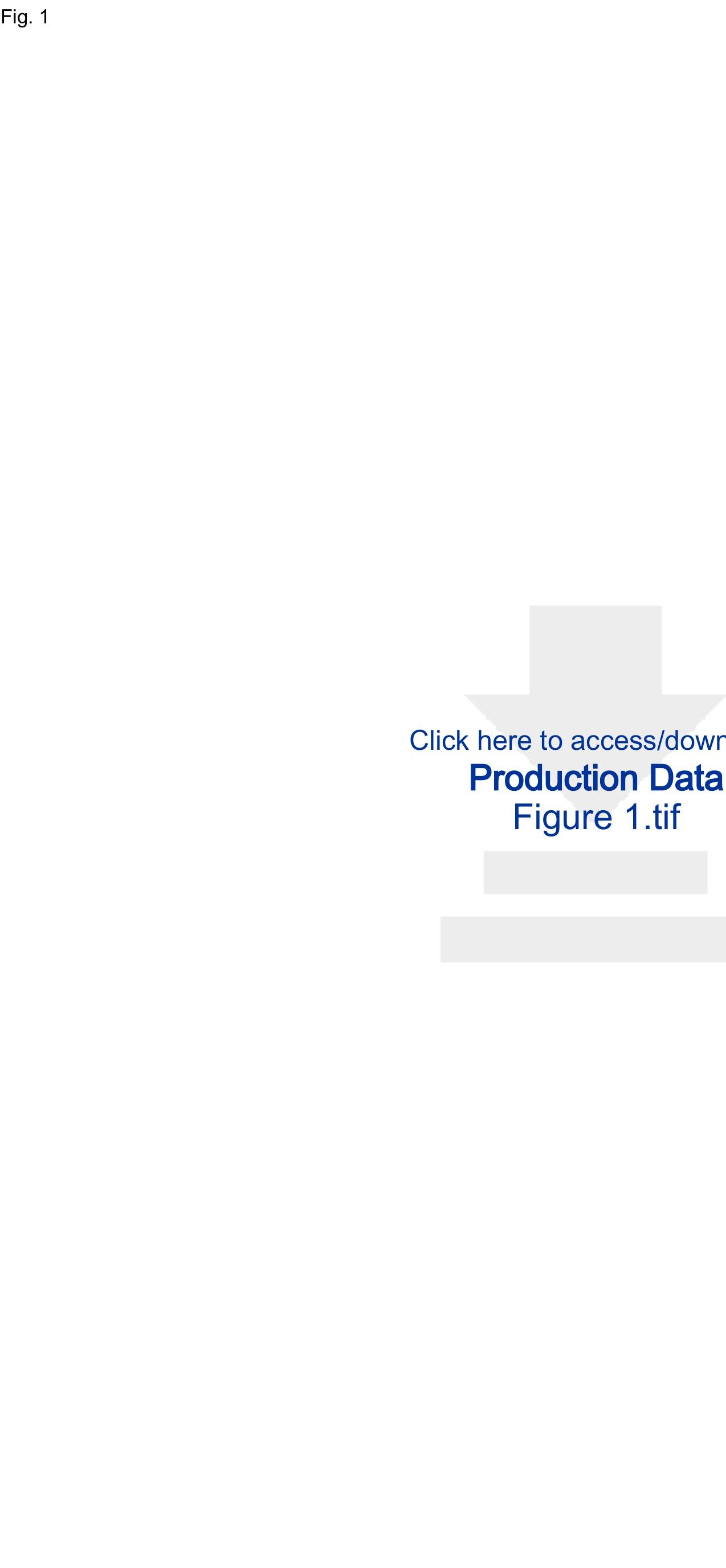
Fig. 2

\section{Click here to access/download \\ Production Data Figure 2.tif$$
\text { . }
$$

(a)

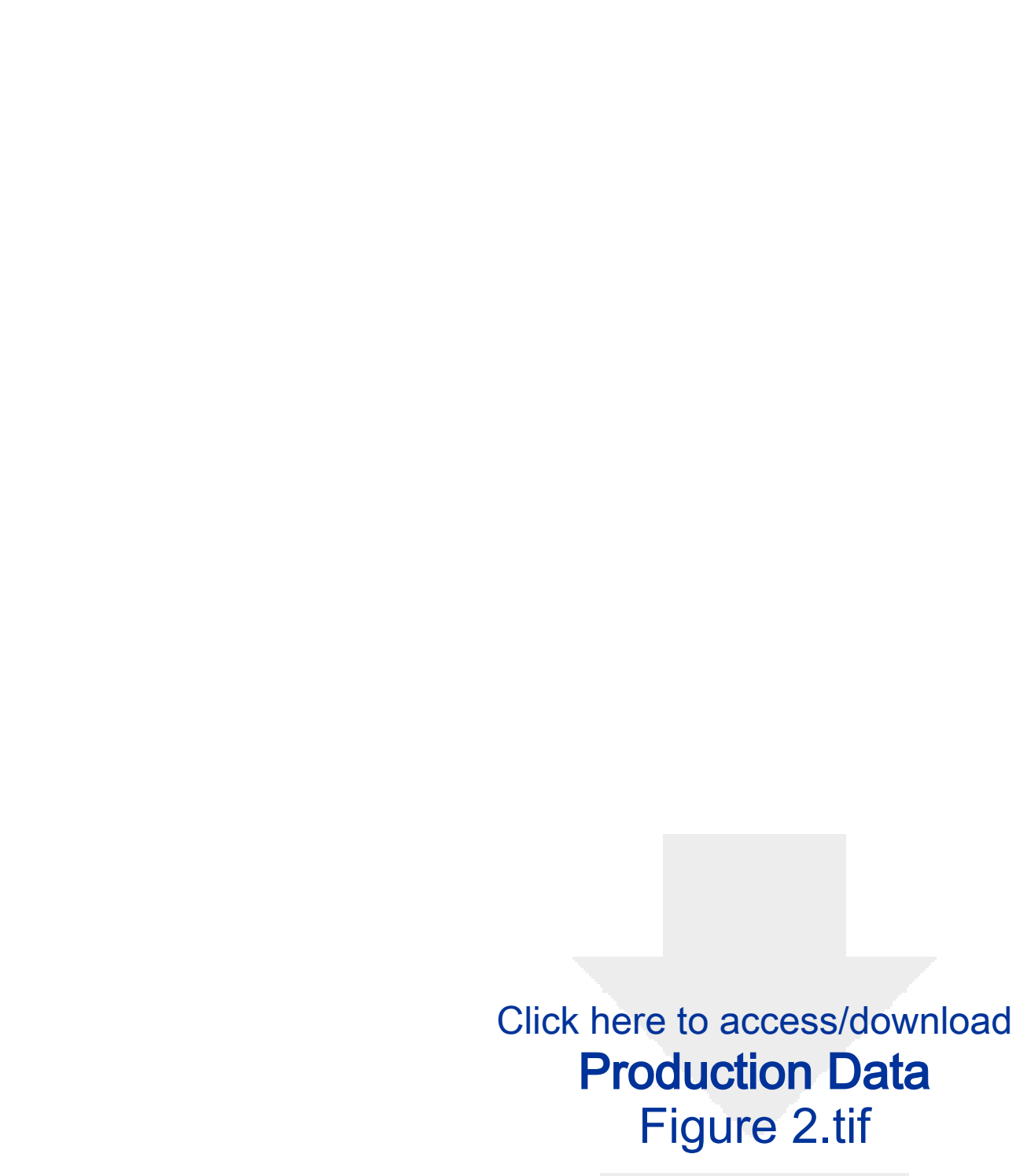

.

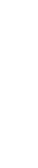

(1) 
Fig. 3

\section{Click here to access/download
Production Data \\ Click here to access/download
Production Data

\author{
Figure 3.tif

\begin{abstract}
Figure 3.4 .
\end{abstract}

(
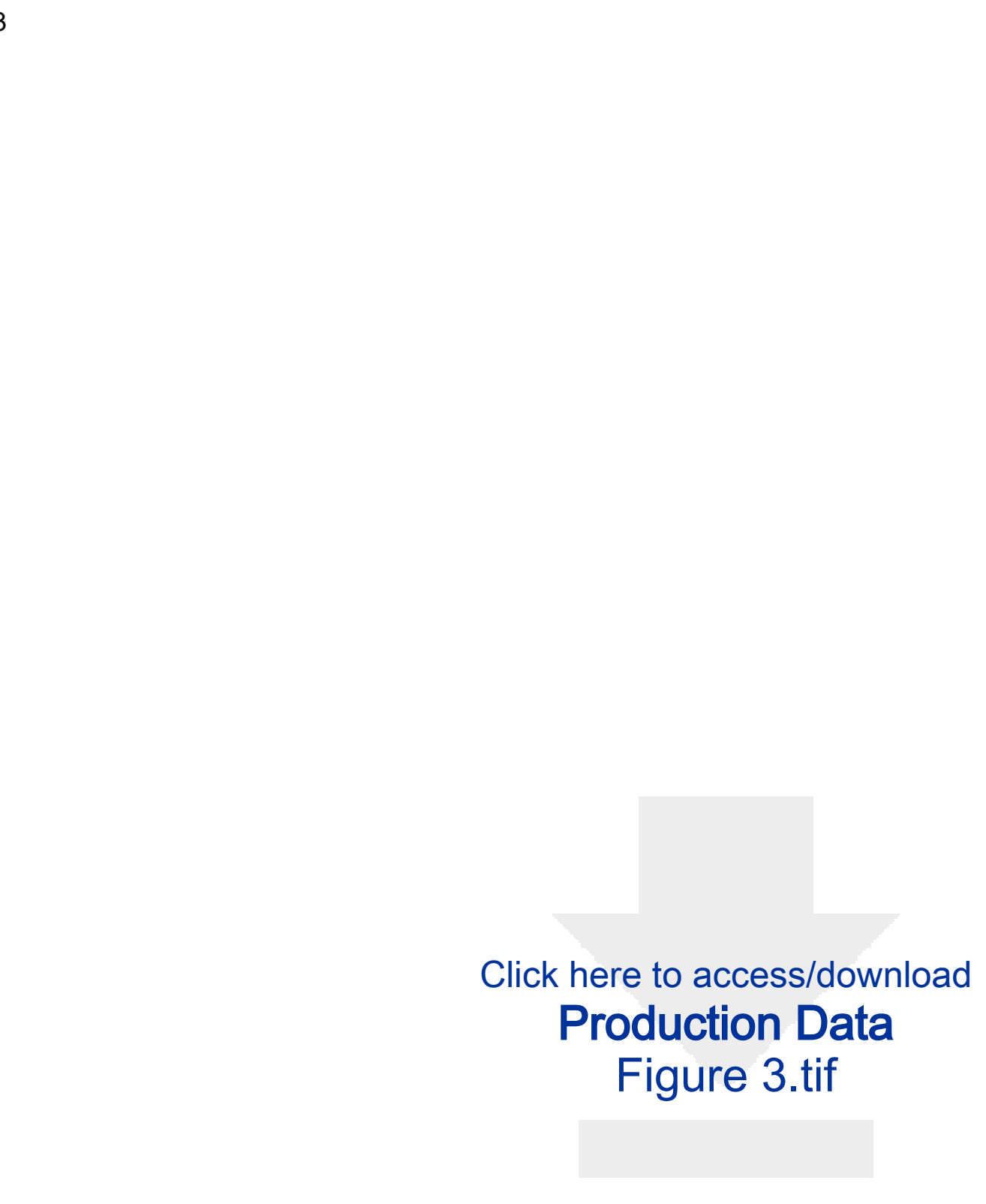

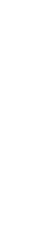


Fig. 4
\[ \begin{array}{c}\text { Click here to access/download } \\ \text { Production Data }\end{array} \]

Fig. 4
\[ \begin{array}{c}\text { Production Data } \\ \text { Click here to access/downlo }\end{array} \]

Figure 4.tif

Fig. 4 Production Data
Click here to access/downlo

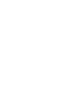

Fig. 4
\[ \begin{array}{c}\text { Click here to access/download } \\ \text { Production Data } \\ \text { Figure 4.tif }\end{array} \]

Figure 4 .tif

Figure 4.tif

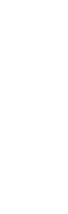


Fig. 5

\section{Click here to access/download \\ Production Data Figure 5.tif

a

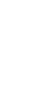
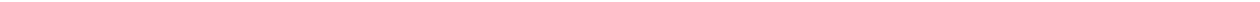
Fig. 6

6

.

- 
Fig. 7

\section{Click here to access/download
Production Data \\ Click here to access/download
Production Data
Figure 7.tif Production Data
Figure 7.tif Production Data
Figure 7.tif}

.

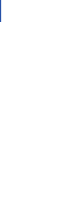

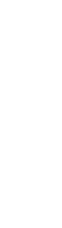

(1) 
Click here to access/download

\section{Production Data Graphic of abstract.tif}

